\title{
Falling balls in a viscous fluid with contact: Comparing numerical simulations with experimental data
}

\author{
HENRY VON WAHL ${ }^{*}, \dagger$ \\ Thomas RichteR ${ }^{\dagger} \quad$ Stefan Frei ${ }^{\ddagger}$ \\ Thomas Hagemeier $§$
}

18th November 2020

\begin{abstract}
We evaluate a number of different finite element approaches for fluid-structure (contact) interaction problems against data from physical experiments. For this we take the data from experiments by Hagemeier [Mendeley Data, DOI: 10.17632/mf27c92nc3.1]. This consists of trajectories of single particles falling through a highly viscous fluid and rebounding off the bottom fluid tank wall. The resulting flow is in the transitional regime between creeping and turbulent flows. This type of configuration is particularly challenging for numerical methods due to the large change of the fluid domain and the contact between the wall and particle. In the numerical simulations we consider both rigid body and linear elasticity models for the falling particles. In the first case, we compare results obtained with the well established Arbitrary Lagrangian Eulerian (ALE) approach and a moving domain CutFEM method together with a simple and common approach for contact avoidance. For the full fluid-structure interaction (FSI) problem with contact, we use a fully Eulerian approach in combination with a unified FSI-contact treatment using Nitsche's method. For higher computational efficiency we use the geometrical symmetry of the experimental set up to reformulate the FSI system into two spatial dimensions. Finally, we show full three dimensional ALE computations to study the effects of small perturbations in the initial state of the particle to investigate deviations from a perfectly vertical fall observed in the experiment. The methods are implemented in open-source finite element libraries and the results are made freely available to aide reproducibility.
\end{abstract}

\section{Introduction}

Flows containing particles, i.e., particulate flows or particles settling in a fluid, have many industrial and biological applications. Examples range from the transport of platelets in blood flows to the sedimentation of sand in pipelines.

We shall consider single elastic spherical particles falling freely in a viscous fluid and rebounding off the bottom wall of the fluid domain at Reynolds numbers in the transitional regime between creeping and turbulent flows. The multiphase and fluid-structure interaction (FSI) problem with solid contact posed by the settling in the fluid and rebounding off a wall is challenging from both an analytical and numerical perspective.

From the theoretical point of view, the correct model for the transition to contact with the bottom wall is not yet fully understood. In the case where a rigid solid is assumed, most flow models lead to results contradicting real world observations. For example, if a creeping flow is assumed, such that the linear Stokes equations are applicable, then contact can only occur under singular forces, c.f. [Bre61]. When the nonlinear incompressible Navier-Stokes together with no-slip boundary conditions are taken for the fluid model, then contact cannot occur and it is impossible to release contact [Fei03b]. This can however be overcome,

\footnotetext{
*Corresponding author: henry.vonwahl@ovgu.de

${ }^{\dagger}$ Insitut für Analysis und Numerik, Otto-von-Guericke Universität, Universitätsplatz 2, D-39106 Magdeburg

${ }_{\ddagger}^{\ddagger}$ Department of Mathematics and Statistics, University of Konstanz, Universitätsstraße 10, D-78457 Konstanz

§Institut für Strömungstechnik und Thermodynamik, Otto-von-Guericke Universität, Universitätsplatz 2, D-39106 Magdeburg
} 
if the boundary condition is modified to a free-slip condition [GHW15], the rough nature of the surface is taken into account [GH14] or the fluid is taken to be compressible [Fei03a]. If the solid model is changed to take the elasticity of the body into account, then it is currently assumed, that even with perfectly smooth boundaries and incompressibility, rebounding without contact can occur due to the storage of energy in the elastic solid [DSH86; GH16; HT09]. This has been refined recently (in the Stokes setting) [Gra+20], where it has been shown, that internal storage of energy is not sufficient, but that additionally a change in the "flatness" is necessary to achieve physically meaningful rebound without topological contact.

For numerical methods, the challenge lies in the discretisation of the resultant FSI system [Ric17]. It consists of a free boundary value problem with a moving interface. The most well established method for this is the Arbitrary Lagrangian Eulerian (ALE) approach [DGH82]. This approach leads to very efficient and accurate computations in situations where the method is usable. However, its usage limited as it breaks down when deformations with respect to the reference configuration become too large and when contact occurs [FRW16]. To deal with large deformations, overlapping mesh techniques have been developed [JLL15]. Here the background fluid domain and the region around the structure are meshed separately so that the fluid-solid interface is resolved. The two meshes are then coupled using unfitted approaches. This then allows a hybrid approach, where the solid and the near fluid are treated using the ALE framework, while the remaining fluid is treated in Eulerian coordinates [SAW19]. To overcome both large deformations and contact, fully Eulerian approaches have lately become the focus of research. In the case of rigid bodies, a number of different approaches have been considered. For example, based on fictitious domain methods using Lagrange multipliers [Glo+99], XFEM type approaches [CFL13; CF15] and most recently CutFEM approaches using Nitsche's method [BFM19; WRL20]. Here a major issue remains that of achieving a realistic rebound effect, since an artificial contact/lubrication force is added to the equation governing the motion of the solid to prevent overlap of the solid regions [Glo+99]. Nevertheless, topological changes appear to be unproblematic for the CutFEM type approaches [LO19].

Considering full fluid-structure interactions, immersed approaches have become popular in recent years [HH03; LCB06; GW08; BF14]. Here the fluid and the solid are treated in their natural Eulerian and Lagrangian coordinate systems, respectively, and the sub-domains are meshed separately. The two meshes are then coupled by means of Nitsche's method [BF14; HH03] or using Lagrange multipliers [Baa01; LCB06; GW08]. Another possibility to handle large deformations and contact are fully Eulerian approaches, where both the solid and fluid equations are formulated in the Eulerian coordinate framework, which simplifies the coupling within monolithic algorithms [Dun06; CMM08; Ric13; Fre16; HP17]. All these approaches are however relatively new and require further development with respect to accuracy and robustness.

The aforementioned methods have been applied to different test cases for numerical validation and a priori error estimates are also available in most cases. Established benchmarks for fluid structure interaction problems such as [TH06] completely avoid contact, since the methods which handle contact remain relatively new. For rigid body motion, most numerical studies are interpreted qualitatively or compared to artificial, analytically derived solutions. Especially in the cases where artificial forces are introduced in order to avoid contact of rigid solids, real validation is near impossible, as this introduces model parameters for which there is no a priori knowledge on a good choice. However, a number of FSI methods have recently become available that are able to resolve contact [Age+19; ASW19; Bur+19; BFF20; ZAV20]. This then raises the question of how well the different modelling and discretisation approaches depict the behaviour of contact and rebound observed in physical experiments.

In this work, we take recently published data from experiments where different solid spherical particles were allowed to settle in a viscous fluid [HTR20; Hag20]. We then use a rigid-body ALE, a rigid-body Eulerian CutFEM and a fully Eulerian FSI approach to each simulate the scenarios presented by the physical experiments. This aims to show the validity and the applicability of these different approaches to the different aspects/problems posed by this process. Furthermore, we will illustrate how spatially reduced models are able to capture the behaviour in comparison to full three dimensional computations. To the best of our knowledge, there is currently no comparable benchmark which considers such a multiphase flow/ fluid structure interaction problem with contact which is validated against experimental data.

The remainder of this paper is structured as follows. In section 2 we describe the considered problem, that is a description of the physical experiment, the mathematical models used to described the experiment, 
the specific set-ups we will simulate and the quantities used to compare the numerical simulations with the experimental data. Section 3 then briefly covers the reduced formulation we apply to increase the computational efficiency in our numerical methods. The numerical computations are then presented in section 4; we present the details of the different numerical approaches in subsection 4.1 and the results are then presented in subsection 4.2. We discuss the conclusions from these results in section 5 and consider the aspect which remain open. Furthermore, we define and compute two simplified set-ups in Appendix A, designed to help others reproduce the presented computational results.

\section{Description}

We describe the experimental set-up used to gather the data, the mathematical model we will use to reproduce the behaviour observed in the experiments and we define relevant quantities used to compare the results quantitatively.

\subsection{Physical Experiment}

The experiments in [HTR20] capture the settling and impact process of spherical particles with different size and density in a cylindrical tank. The later contains a liquid mixture consisting of glycerine and water at equal volume fractions. At the bottom of the cylindrical tank, which is made of acrylic glass for optical access, a massive steel anvil serves as impact object. Moreover, the cylinder is surrounded by a rectangular container, filled with refractive index matching liquid, to compensate for optical distortion coming from the curved cylinder walls. The filling level allows the observation of the particle settling along a vertical distance of 140 to $160 \mathrm{~mm}$, depending on the particle size. Initially, each particle is held in place and submerged in the liquid by a vacuum tweezer. The particle is then released by switching off the vacuum pump. The particle is tracked during the settling process and the impact on the steel anvil, including the rebound using a high-speed CMOS-camera. This acquires shadow images at a frame rate of 1000 frames per second and a scale factor of 8.89 pixel per mm. An image processing algorithm coded in MATLAB yields the in-plane particle coordinates as function of time and allows to extract the instantaneous particle settling velocity.

The resulting data is available via Mendeley Data [Hag20]. This data is the basis for our comparison and validation of the numerical code.

\subsection{Mathematical model}

We consider a bounded domain $\Omega \in \mathbb{R}^{d}$, with $d \in\{2,3\}$, over a finite, non-empty, time interval $\left[0, T_{\text {end }}\right]$. This is divided into a $d$-dimensional fluid region $\mathcal{F}$, a $d$-dimensional solid $\mathcal{S}$ and a $d$-1-dimensional interface $\mathcal{I}$ dividing the solid and fluid regions. For these we have $\Omega=\mathcal{F} \dot{\cup} \mathcal{I} \dot{\mathcal{S}}$.

Fluid model. In the time dependent fluid region $\mathcal{F}(t)$, we consider the incompressible Navier-Stokes equations. Find a velocity $\boldsymbol{u}$ and a pressure $p$ such that

$$
\begin{aligned}
\rho_{f}\left(\partial_{t} \boldsymbol{u}+(\boldsymbol{u} \cdot \nabla) \boldsymbol{u}\right)-\operatorname{div} \boldsymbol{\sigma}(\boldsymbol{u}, p) & =0 \\
\operatorname{div}(\boldsymbol{u}) & =0
\end{aligned}
$$

with the non-symmetric stress tensor

$$
\boldsymbol{\sigma}(\boldsymbol{u}, p)=\mu_{f} \nabla \boldsymbol{u}-p \mathrm{Id}
$$

where $\mu_{f}=\rho_{f} \nu_{f}$ is the fluid's dynamic viscosity, $\nu_{f}$ is the kinematic viscosity and $\rho_{f}$ is the fluid's density. Appropriate boundary conditions to complete this system will be discussed later. Note that we use bold face letters to denote vector/matrix valued quantities while regular faced letters denote scalar objects. 
Elastic solid and fluid-structure interaction. We consider a linear elastic solid model in $\mathcal{S}$ for the solid displacement $\boldsymbol{d}$ and the solid velocity $\dot{\boldsymbol{d}}$, given by

$$
\rho_{s} \partial_{t} \dot{\boldsymbol{d}}-\operatorname{div} \boldsymbol{\sigma}_{s}(\boldsymbol{d})=\left(\rho_{s}-\rho_{f}\right) g, \quad \dot{\boldsymbol{d}}=\partial_{t} \boldsymbol{d},
$$

with the acceleration due to gravity $g=-9.807 \mathrm{~m} \mathrm{~s}^{-2}$ and the Cauchy stress tensor $\boldsymbol{\sigma}_{s}$ defined by

$$
\boldsymbol{\sigma}_{s}(\boldsymbol{d})=2 \mu_{s} E(\boldsymbol{d})+\lambda_{s} \operatorname{tr}(E(\boldsymbol{d})) I, \quad E(\boldsymbol{d})=\frac{1}{2}\left(\nabla \boldsymbol{d}+\nabla \boldsymbol{d}^{T}\right)
$$

where $\mu_{s}$ and $\lambda_{s}$ denote the Lamé parameters. Note that we have subtracted the fluid gravitational force $\rho_{f} g$ on the right-hand side of the solid equation to be consistent with the equations for a rigid solid presented in the following paragraph. Alternatively this could be added on the right-hand side of the fluid equations.

Solid and fluid are coupled by means of no-slip coupling conditions on $\mathcal{I}$

$$
\boldsymbol{u}=\dot{\boldsymbol{d}}, \quad \boldsymbol{\sigma}(\boldsymbol{u}, p) \boldsymbol{n}=\boldsymbol{\sigma}_{s}(\boldsymbol{d}) \boldsymbol{n} .
$$

The current position of the interface $\mathcal{I}(t)$ is determined by the displacement variable $\boldsymbol{d}$.

Rigid solid. As the solid materials we will consider are relatively hard, the consideration of a rigid solid yields a good approximation of the FSI dynamics, at least up to the moment when the solid comes close to the lower wall. The movement of the solid is governed by Newton's second law of motion. Let $\boldsymbol{c}_{\mathcal{S}}(t)$ be the centre of mass of the solid $\mathcal{S}$. Since we will consider spherical particles, this is then governed by

$$
\frac{\mathrm{d}^{2}}{\mathrm{~d} t^{2}} \boldsymbol{c}_{\mathcal{S}}(t) \cdot m_{\mathcal{S}}=\boldsymbol{f}_{s}
$$

where $m_{\mathcal{S}}$ is the mass of the solid and $\boldsymbol{f}_{s}$ are the forces acting on the solid in the horizontal and vertical directions. For simplicity, we assume that the horizontal forces are negligible. The vertical forces are then the gravitational pull, buoyancy and the viscous drag

$$
\boldsymbol{f}_{s}=\left(\begin{array}{c}
0 \\
0 \\
m_{\mathcal{S}} g-\operatorname{vol}(\mathcal{S}) \rho_{f} g+\boldsymbol{F}_{3}
\end{array}\right)
$$

where $\operatorname{vol}(\mathcal{S})$ is the volume of the solid and $\boldsymbol{F}_{3}$ the viscous drag force in the vertical direction. This is the third component of

$$
\boldsymbol{F}=\int_{\mathcal{I}} \boldsymbol{\sigma}(\boldsymbol{u}, p) \boldsymbol{n} \mathrm{d} s .
$$

Note that we added the effects of buoyancy in (2.5). This would be naturally included in $\boldsymbol{F}$ if we added body force $\rho_{f} g \nabla \boldsymbol{x}_{d}$ to the right-hand side of the fluid equation (2.1a). However since this would only affect the pressure, it is sufficient to consider the homogeneous equation (2.1a) and add the effect of buoyancy to (2.5). Furthermore, this approach is more accurate in our case, since only pressure-robust methods are able to reflect this effect of gradient contributions in the forcing term on the pressure exactly on the numerical level [Joh+17].

As a result of us neglecting horizontal movement of the solid, (2.4) becomes a scalar ODE. We can also simplify the terms in (2.5), so that in total we come to the equation

$$
\frac{\mathrm{d}}{\mathrm{d} t} \boldsymbol{v}_{\mathcal{S}, 3}(t)=\frac{\rho_{s}-\rho_{f}}{\rho_{s}} g+\frac{\boldsymbol{F}_{3}}{\operatorname{vol}(\mathcal{S}) \rho_{s}}
$$

where $\boldsymbol{v}_{\mathcal{S}}(t)=\frac{\mathrm{d}}{\mathrm{d} t} \boldsymbol{c}_{\mathcal{S}}(t)$ is the solid's velocity.

The solid's motion couples back to the fluid equations through the boundary condition at the interface $\mathcal{I}$, by requiring continuity of the velocity, i.e.,

$$
\left.\boldsymbol{u}\right|_{\mathcal{I}}=\boldsymbol{v}_{\mathcal{S}}=\frac{\mathrm{d}}{\mathrm{d} t} \boldsymbol{c}_{\mathcal{S}}(t) .
$$

We note that this model neglects rotational effects. As will be shown below, this does not have a major impact on the quality of the resulting approximations. 


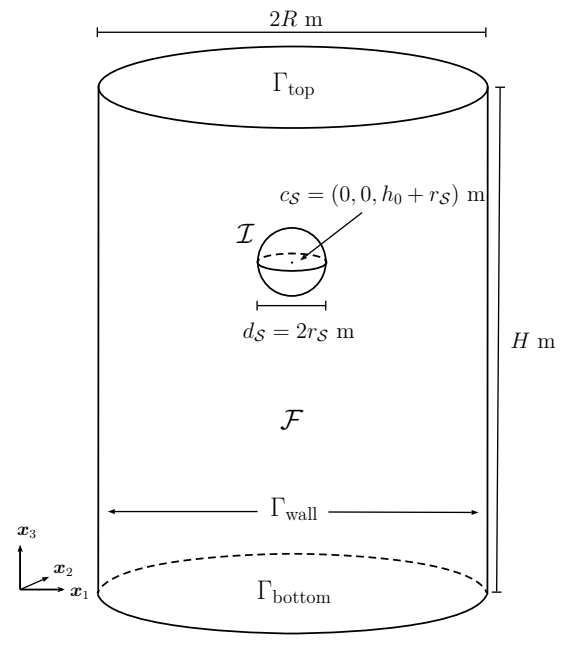

(a) Three dimensions

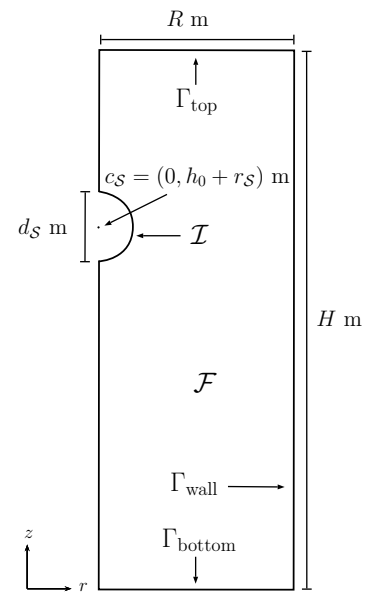

(b) Roationally reduced

Figure 1.: The initial spatial configuration.

\subsection{Domain Description}

Since we consider balls of different sizes, we shall keep the domain description general. The background domain is a cylinder $\Omega=\left\{\boldsymbol{x}=(x, y, z)^{T} \in \mathbb{R}^{3} \mid x^{2}+y^{2}<R^{2}, 0<z<H\right\}$ for a given radius $R$ and a given height $H$. At $t=0$ the solid domain is described by $\mathcal{S}(0)=\left\{\boldsymbol{x}=(x, y, z)^{T} \in \mathbb{R}^{3} \mid x^{2}+y^{2}+\left(z-\left(h_{0}+r_{\mathcal{S}}\right)\right)^{2}<\right.$ $\left.r_{\mathcal{S}}^{2}\right\}$ for a given ball radius $r_{\mathcal{S}}$ and an initial height of the bottom of the ball $h_{0}$. Accordingly, the volume of the solid is given by $\operatorname{vol}(\mathcal{S})=4 \pi r^{3} / 3$. An illustration of this can be seen in Figure 1a.

\subsubsection{Boundary conditions}

We denote the top boundary $(z=H)$ of the cylinder as $\Gamma_{\text {top }}$, the bottom boundary $(z=0)$ as $\Gamma_{\text {bottom }}$ and the side of the cylinder $\left(x^{2}+y^{2}=R^{2}\right)$ as $\Gamma_{\text {wall }}$.

On the interface $\mathcal{I}$ between the solid and the fluid, the Dirichlet boundary condition is given by the continuity of the velocity, see (2.3) and (2.8). On the wall and bottom boundaries $\Gamma_{\text {bottom }} \cup \Gamma_{\text {wall }}$ we shall impose homogeneous Dirichlet boundary conditions $\boldsymbol{u}=\mathbf{0}$. In order to approximate the free surface at the top of the water tank $\Omega$, we impose a free-slip boundary condition $\boldsymbol{u}_{3}=0$ at $\Gamma_{\text {top }}$.

\subsection{Material parameters}

The geometrical and material parameters are as follows. The cylindrical tank has a diameter of $110 \mathrm{~mm}$ giving a radius of $R=0.055 \mathrm{~m}$ and has a height of $H=0.2 \mathrm{~m}$. The density, viscosity and surface tension of the highly viscous fluid are $\rho_{F}=1141 \mathrm{~kg} \mathrm{~m}^{-3}, \mu_{F}=0.008 \mathrm{~kg} \mathrm{~m}^{-1} \mathrm{~s}^{-1}$ and $\gamma_{F}=0.064 \mathrm{~N} \mathrm{~m}^{-2}$, respectively.

\subsubsection{PTFE6}

We consider a polytetrafluorethylen/teflon (PFTE) particle with diameter $d_{\mathcal{S}}=6.0 \mathrm{~mm}$, i.e., $r_{\mathcal{S}}=0.003 \mathrm{~m}$., and a weight of $0.24 \mathrm{~g}$, which yields a particle density of $\rho_{\mathrm{PTFE}}=2122 \mathrm{~kg} \mathrm{~m}^{-3}$. For the case of an unconfined free fall, we calculated a terminal velocity of $v_{T}=0.38 \mathrm{~m} \mathrm{~s}^{-1}$ and a relaxation time of $t_{R}=0.1 \mathrm{~s}$ according to $[\mathrm{Man}+15]$. The corresponding terminal Reynolds number is $R e_{\mathrm{PTFE}}=333$.

Young's modulus and Poisson ratio of the PTFE ball are about $670 \mathrm{MPa}=6.7 \cdot 10^{8} \mathrm{~kg} \mathrm{~m}^{-1} \mathrm{~s}^{-2}{ }^{1}$ and

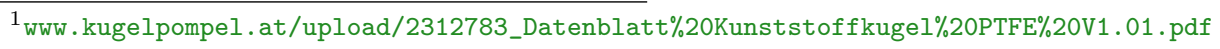




\begin{tabular}{lcccccccc}
\hline \multirow{2}{*}{ Experiment } & \multicolumn{4}{c}{ Geometry } & & \multicolumn{3}{c}{ Boundary Conditions } \\
\cline { 2 - 4 } \cline { 7 - 8 } & $\mathrm{R}(\mathrm{m})$ & $\mathrm{H}(\mathrm{m})$ & $r_{\mathcal{S}}(\mathrm{m})$ & $h_{0}(\mathrm{~m})$ & & $\Gamma_{\text {wall }} \cup \Gamma_{\text {bottom }}$ & $\Gamma_{\text {top }}$ & $\mathcal{I}$ \\
\hline PTFE6 & \multirow{2}{*}{0.055} & 0.2 & 0.003 & 0.1616616 & & $\boldsymbol{u}=0$ & $\boldsymbol{u}_{d}=0$ & $\boldsymbol{u}=\boldsymbol{v}_{\mathcal{S}}$ \\
Rubber22 & & & 0.011 & 0.1461203 & & & & \\
\hline
\end{tabular}

Table 1.: Geometrical parameters of the test cases.

\begin{tabular}{|c|c|c|c|c|c|c|}
\hline \multirow[t]{2}{*}{ Experiment } & & \multicolumn{5}{|c|}{ Material parameters } \\
\hline & $g\left(\mathrm{~m} \mathrm{~s}^{-2}\right)$ & $\mu_{f}\left(\mathrm{~kg} \mathrm{~m}^{-1} \mathrm{~s}^{-1}\right)$ & $\rho_{f}\left(\mathrm{~kg} \mathrm{~m}^{-3}\right)$ & $\rho_{s}\left(\mathrm{~kg} \mathrm{~m}^{-3}\right)$ & $\lambda_{s}\left(\mathrm{~kg} \mathrm{~m}^{-1} \mathrm{~s}^{-2}\right)$ & $\mu_{s}\left(\mathrm{~kg} \mathrm{~m}^{-1} \mathrm{~s}^{-2}\right)$ \\
\hline $\begin{array}{l}\text { PTFE6 } \\
\text { Rubber22 }\end{array}$ & -9.807 & 0.008 & 1141 & $\begin{array}{l}2122 \\
1361\end{array}$ & $\begin{array}{l}2.63870 \cdot 10^{9} \\
3.33289 \cdot 10^{9}\end{array}$ & $\begin{array}{l}2.29452 \cdot 10^{8} \\
6.66711 \cdot 10^{5}\end{array}$ \\
\hline
\end{tabular}

Table 2.: Summary of the benchmark set-up in standard units.

$\nu_{s}=0.46^{2}$, respectively, which yields the Lamé parameters $\lambda_{s} \approx 2.63870 \cdot 10^{9} \mathrm{~kg} \mathrm{~m}^{-1} \mathrm{~s}^{-2}$, and $\mu_{s} \approx$ $2.29452 \cdot 10^{8} \mathrm{~kg} \mathrm{~m}^{-1} \mathrm{~s}^{-2}$. The spatial parameters are summarised in Table 1 while the used material parameters are summarised in Table 2.

\subsubsection{Rubber 22}

We consider a rubber sphere. This has a diameter of $d_{\mathcal{S}}=22 \mathrm{~mm}$ and a weight of $7.59 \mathrm{~g}$, combining for an effective density of $\rho_{\text {Rubber }}=1361 \mathrm{~kg} \mathrm{~m}^{-3}$. The unconfined free fall characteristics are $v_{T}=0.35 \mathrm{~m} \mathrm{~s}^{-1}$, $t_{R}=0.31 \mathrm{~s}$ and $R e_{\text {Rubber }}=1109$.

Chemical analysis of the material suggests this to be hydrogenated nitrile rubber (HNBR). Young's modulus and Poisson ratio of this are approximately $1.7-2.07 \cdot 10^{1} \mathrm{MPa}=1.7 \cdot 10^{6}-2.1 \cdot 10^{7} \mathrm{~kg} \mathrm{~m}^{-1} \mathrm{~s}^{2}{ }^{3}$ and $\nu_{s}=$ 0.4999 [AMR04]. With $E_{s}=2 \cdot 10^{6}$, we have the Lamé parameters $\lambda_{s}=3.33289 \cdot 10^{9} \mathrm{~kg} \mathrm{~m}^{-1} \mathrm{~s}^{-2}$ and $\mu_{s}=6.66711 \cdot 10^{5} \mathrm{~kg} \mathrm{~m}^{-1} \mathrm{~s}^{-2}$. These parameters are again summarised in Table 1 and Table 2 .

\subsection{Quantities of interest}

We will use the following quantities to compare our numerical results with each other and with the experimental data.

$t_{*} \quad$ Let $t_{0}=\left.t\right|_{\boldsymbol{c}_{\mathcal{S}}=h_{0}}$ be the time at which the centre of mass is at $h_{0}$, i.e., when the ball has traveled $r_{\mathcal{S}}$ vertically. We define $t_{*}$ as the time after release when $\operatorname{dist}\left(\mathcal{I}, \Gamma_{\text {bottom }}\right)=d_{\mathcal{S}}$ relative to $t_{0}$, i.e., for PTFE6 $t_{*}=\left.t\right|_{c_{\mathcal{S}}=(0,0,0.009)}-t_{0}$ and for Rubber22 $t_{*}=\left.t\right|_{c_{\mathcal{S}}=(0,0,0.033)}-t_{0}$.

$v_{*} \quad$ The velocity of the ball in the $z$-direction at $t=t_{*}+t_{0}$.

$f_{*} \quad$ The vertical component of the force $\boldsymbol{F}$ acting on the ball at time $t_{*}+t_{0}$.

$t_{\text {cont }}$ The time of the first solid contact relative to $t_{0}$.

$t_{\text {jump }}$ The time between contact and the second change in direction is realised, i.e., the amount of time the balls travels upwards after the first contact.

$d_{\text {jump }}$ The maximum of $\operatorname{dist}\left(\mathcal{I}, \Gamma_{\text {bottom }}\right)$ after contact, i.e., the size of the bounce.

An illustration of how these quantities are defined can be seen in Figure 2.

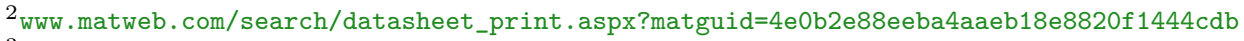

3 eriks.de/content/dam/de/pdf/downloads/dichtungen/o-ringe/ERIKS_Technisches-Handbuch-0-Ringe_de.pdf p. 20.
} 


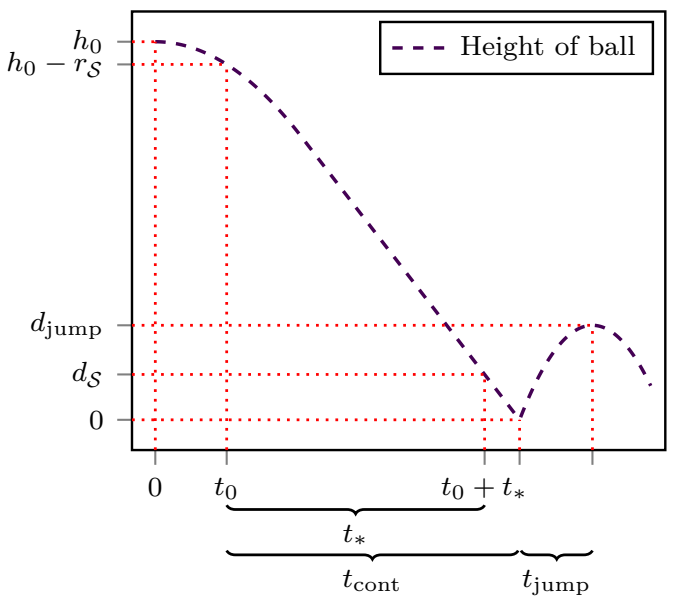

Figure 2.: Illustration for the definitions if the quantities of interest.

\begin{tabular}{lccccc}
\hline Experiment & $t_{*}^{\exp }$ & $v_{*}^{\exp }$ & $t_{\text {cont }}^{\exp }$ & $t_{\text {jump }}^{\exp }$ & $d_{\text {jump }}^{\exp }$ \\
\hline PTFE6 & 0.516403 & -0.330987 & 0.492232 & 0.027918 & 0.002212 \\
Rubber22 & 0.469137 & -0.309301 & 0.544021 & 0.089492 & 0.004149 \\
\hline
\end{tabular}

Table 3.: Quantities of interest as established from the experimental data.

Remark (Choice of reference values.). In the experiments, we observed that the balls do not immediately start to fall after they are released. The settling process starts with some distance to the liquid surface, but in closest vicinity of the vacuum cup. In general, particles experience an increased drag force when they are moving towards or away from a solid wall and free fluid-surfaces. Accordingly, the early stage of the settling process here is dominated by an increased drag force coming from the vacuum cup and the liquid surface.

Due to the rather slow motion of the sphere at the beginning, the moment of release cannot be defined well. To be able to compare the numerical results with the experimental data, we therefore defined the above quantities relative to the point in time at which the the ball has traveled the distance of the balls radius. Furthermore, since we have no measurement of the drag force in in the experiment, $f_{*}$ will only be used to compare the computations directly.

To establish $v_{*}, t_{\text {cont }}, d_{\text {cont }}$ and $d_{\text {jump }}$ from the experimental data we interpolate the height data using a spline of order 3. This spline is then evaluated to establish the time at which $\boldsymbol{c}_{\mathcal{S}}=\left(0,0,3 r_{\mathcal{S}} / 2\right)$, the time of contact as well as the time and height of the jump. The velocity is then taken as the first derivative of the spline representing the height. The resulting reference values for the quantities of interest are summarised in Table 3.

The experimental study [HTR20] was conducted, so that the horizontal displacement of the particles was minimal. The data set we will compare against shows a maximal horizontal displacement of less than $2.0 \mathrm{~mm}$ and $7.5 \cdot 10^{-1} \mathrm{~mm}$ in the PTFE6 and Rubber22 cases respectively. This compares with the mean over time of the maximal deviation in the centre location between experiment repetitions of $1.9 \cdot 10^{-1} \mathrm{~mm}$ and $1.4 \cdot 10^{-1} \mathrm{~mm}$ for the PTFE6 and Rubber22 cases respectively. However, the experiment is only able to give the projection of the horizontal displacement onto the $x$ - $z$-plane. Thus is is not possible to detect the true horizontal motion. Note that we have any horizontal motion this in the computation of the reference values. However, since overall the horizontal deflection is small, we consider this to be reasonable for the present purpose. 


\section{Reduced model}

The set-up described in subsection 2.3 is symmetric with respect to rotation, if viewed in cylindrical coordinates. The experimental data presented in [HTR20; Hag20] shows a rotational component in the motion of the solid and it also shows a small deflection of the center of mass $c_{\mathcal{S}}$ from the $z$-axis. However, these effects are small and since the material parameters are such that the resulting flow is the low to intermediate Reynolds-number regime [HTR20], we assume that the solution is described sufficiently well by a rotationally symmetric flow in cylinder coordinates. We use this in order to obtain a two-dimensional reduced formulation which is computationally cheaper compared to full three dimensional computations. In subsubsection 4.2.3 we will also present a fully resolved three dimensional simulation to have a closer look at these rotational effects. To distinguish between the full three dimensional domains and the reduced two dimensional domain, we shall denote objects stemming from the three-dimensional set-up with a superscript " $3 d$ " if there is a potential for ambiguity. For the sake of readability, object based on the reduced two-dimensional set-up will not have a special notation.

With the spaces $\boldsymbol{V}^{3 d}=\left\{\boldsymbol{v} \in \mathcal{H}^{1}\left(\mathcal{F}^{3 d}\right)|\boldsymbol{v}|_{\mathcal{I}^{3 d}}=\boldsymbol{u}_{\mathcal{S}^{3 d}},\left.\boldsymbol{v}\right|_{\Gamma_{\text {wall }}^{3 d} \cup \Gamma_{\text {bottom }}^{3 d}}=0\right.$ and $\left.\left.\boldsymbol{v}_{z}\right|_{\Gamma_{\text {top }}^{3 d}}=0\right\} \subset\left[\mathcal{H}^{1}\left(\mathcal{F}^{3 d}\right)\right]^{3}$ and $Q^{3 d}=\mathcal{L}_{0}^{2}\left(\mathcal{F}^{3 d}\right)$, the weak formulation of the Navier-Stokes equation (2.1) is as follows:

$$
\begin{gathered}
\text { Find }(\boldsymbol{u}, p) \in \boldsymbol{V}^{3 d} \times Q^{3 d} \text { such that for all }(\boldsymbol{v}, q) \in \boldsymbol{V}^{3 d} \times Q^{3 d} \text { it holds } \\
A_{f}^{3 d}(\boldsymbol{u}, p ; \boldsymbol{v}, q):=m^{3 d}\left(\partial_{t} \boldsymbol{u}, \boldsymbol{v}\right)+a^{3 d}(\boldsymbol{u}, \boldsymbol{v})+c^{3 d}(\boldsymbol{u}, \boldsymbol{u}, \boldsymbol{v})+b^{3 d}(\boldsymbol{v}, p)+b^{3 d}(\boldsymbol{u}, q)=0
\end{gathered}
$$

with the multilinear forms

$$
\begin{aligned}
m^{3 d}(\boldsymbol{u}, \boldsymbol{v}) & =\rho_{f} \int_{\mathcal{F}^{3 d}} \boldsymbol{u} \cdot \boldsymbol{v} \mathrm{d} \boldsymbol{x} & a^{3 d}(\boldsymbol{u}, \boldsymbol{v}) & =\mu_{f} \int_{\mathcal{F}^{3 d}} \nabla \boldsymbol{u}: \nabla \boldsymbol{v} \mathrm{d} \boldsymbol{x} \\
c^{3 d}(\boldsymbol{u}, \boldsymbol{v}, \boldsymbol{w}) & =\rho_{f} \int_{\mathcal{F}^{3 d}}(\boldsymbol{u} \cdot \nabla) \boldsymbol{v} \cdot \boldsymbol{w} \mathrm{d} \boldsymbol{x} & b^{3 d}(q, \boldsymbol{v}) & =-\int_{\mathcal{F}^{3 d}} q \nabla \cdot \boldsymbol{v} \mathrm{d} \boldsymbol{x} .
\end{aligned}
$$

In order to reduce this three dimensional flow problem into a two dimensional flow problem, we rewrite the problem into cylinder coordinates $r, \phi, z$. Rotational symmetry of a solution $\boldsymbol{u}$ means that $\partial_{\phi} \boldsymbol{u}=0$. We use this to rotate the domain into the $r^{+}-z$-plane and transform the weak formulation (3.1) accordingly. Now let $\nabla=\left(\begin{array}{l}\partial_{r} \\ \partial_{z}\end{array}\right)$ and $\Omega=\Omega^{3 d} \cap\left(\mathbb{R}^{+} \times \mathbb{R}\right)$ be the reduced two dimensional computational domain. A sketch of the three dimensional domain transformed into two dimensions can be seen in Figure 1b.

At the symmetry boundary $(r=0)$ the boundary conditions

$$
\boldsymbol{u}_{r}=0, \quad \partial_{r} \boldsymbol{u}_{z}=0
$$

are valid. The reduced spaces are then $\boldsymbol{V}=\left\{\boldsymbol{v} \in \mathcal{H}^{1}(\mathcal{F})|\boldsymbol{v}|_{\mathcal{I}}=\left(\begin{array}{c}0 \\ \boldsymbol{v}_{\mathcal{S} 3}\end{array}\right),\left.\boldsymbol{v}\right|_{\Gamma_{\text {wall }} \cup \Gamma_{\text {bottom }}}=0,\left.\boldsymbol{v}_{z}\right|_{\Gamma_{\text {top }}}=\right.$ 0 and $\left.\left.\boldsymbol{v}_{r}\right|_{r=0}=0\right\} \subset\left[\mathcal{H}^{1}(\mathcal{F})\right]^{2}$ and $Q=\mathcal{L}_{0}^{2}(\mathcal{F})$. The reduced Navier-Stokes problem then reads

Find $(\boldsymbol{u}, p) \in \boldsymbol{V} \times Q$ such that for all $(\boldsymbol{v}, q) \in \boldsymbol{V} \times Q$ it holds

$$
A_{f}(\boldsymbol{u}, p ; \boldsymbol{v}, q):=m\left(\partial_{t} \boldsymbol{u}, \boldsymbol{v}\right)+a(\boldsymbol{u}, \boldsymbol{v})+c(\boldsymbol{u}, \boldsymbol{u}, \boldsymbol{v})+b(\boldsymbol{v}, p)+b(\boldsymbol{u}, q)=0
$$

with the transformed multilinear forms

$$
\begin{array}{rlrl}
m(\boldsymbol{u}, \boldsymbol{v}) & =2 \pi \rho_{f} \int_{\mathcal{F}} r \boldsymbol{u} \cdot \boldsymbol{v} \mathrm{d} \boldsymbol{x} & a(\boldsymbol{u}, \boldsymbol{v}) & =2 \pi \mu_{f} \int_{\mathcal{F}} \nabla \boldsymbol{u}: \nabla \boldsymbol{v}+\frac{1}{r} \boldsymbol{u}_{r} \boldsymbol{v}_{r} \mathrm{~d} \boldsymbol{x} \\
c(\boldsymbol{u}, \boldsymbol{v}, \boldsymbol{w}) & =2 \pi \rho_{f} \int_{\mathcal{F}} r(\boldsymbol{u} \cdot \nabla) \boldsymbol{v} \cdot \boldsymbol{w} \mathrm{d} \boldsymbol{x} & b(q, \boldsymbol{v}) & =-\int_{\mathcal{F}} q\left(\boldsymbol{v}_{r}+r \nabla \cdot \boldsymbol{v}\right) \mathrm{d} \boldsymbol{x}
\end{array}
$$

c.f. [BDM99]. Since the motion of the solid is partially driven by the fluid forces acting on it, we also need to transform these. Let $\left(\boldsymbol{u}^{3 d}, p^{3 d}\right) \in \boldsymbol{V}^{3 d} \times Q^{3 d}$ be a rotationally symmetric solution of (3.1) and $(\boldsymbol{u}, p) \in \boldsymbol{V} \times Q$ the solution of (3.4). We can then transform the weak boundary integral formulations as

$$
\int_{\mathcal{I}^{3 d}} \boldsymbol{\sigma}^{3 d}\left(\boldsymbol{u}^{3 d}, p^{3 d}\right) \boldsymbol{n}^{3 d} \cdot \boldsymbol{v}^{3 d} \mathrm{~d} S^{3 d}=2 \pi \int_{\mathcal{I}} r\left(\mu_{f} \nabla \boldsymbol{u}-\operatorname{Id} p\right) \boldsymbol{n} \cdot \boldsymbol{v} \mathrm{d} S .
$$


The forces $\boldsymbol{F}$ can then be computed by inserting the appropriate non-conforming test-functions $\boldsymbol{v}$ into this functional. Using this, we can compute the motion of the solid as before using (2.7).

\section{Numerical computations}

We give the details of the different numerical approaches applied to attempt to reproduce the observed data and present the results attained with these methods.

The full result data-sets of all methods and the source code to reproduce the rigid body ALE and CutFEM methods can be found in the zenodo repository DOI: 10.5281/zenodo.3989604. This also includes the preparatory examples presented in Appendix A.

\subsection{Discretisations}

We provide the details on the formulations of the different discretisation approaches used.

\subsubsection{Fluid-Rigid Body interaction in Arbitrary Lagrangian-Eulerian coordinates}

To compute the spatially reduced coupled problem we formulate the Navier-Stokes equations in Arbitrary Lagrangian Eulerian (ALE) coordinates [HLZ81] by introducing a reference map

$$
T_{A L E}\left(\boldsymbol{c}_{\mathcal{S}}\right): \mathcal{F} \rightarrow \mathcal{F}\left(\boldsymbol{c}_{\mathcal{S}}\right)
$$

where $\boldsymbol{c}_{\mathcal{S}}$ is solid's centre of mass relative to the bottom boundary and where

$$
\mathcal{F}\left(\boldsymbol{c}_{\mathcal{S}}\right)=\{[0,0.055 \mathrm{~m}] \times[0,0.2 \mathrm{~m}]\} \backslash B_{r_{\mathcal{S}}}\left(\boldsymbol{c}_{\mathcal{S}}\right),
$$

$B_{r_{\mathcal{S}}}\left(\boldsymbol{c}_{\mathcal{S}}\right)$ being the open ball of radius $r$ with midpoint $\boldsymbol{c}_{S}$. As reference domain we set $\mathcal{F}:=\mathcal{F}(0.05 \mathrm{~m})$ where the ball is centred in $\boldsymbol{c}_{\mathcal{S}}=(0,0,0.5)$ such that the mesh distortion is not too extreme when the solid comes close to the lower boundary. The mapping $T_{A L E}\left(\boldsymbol{c}_{\mathcal{S}}\right)$ is given analytically by

$$
\begin{aligned}
T_{A L E}\left(\boldsymbol{c}_{\mathcal{S}} ; r, z\right) & =\left(r, z+\left(\boldsymbol{c}_{\mathcal{S}}-0.05 \mathrm{~m}\right) f_{A L E}(z)\right) \\
f_{A L E}(z) & = \begin{cases}\frac{z}{0.05 \mathrm{~m}-2 r_{\mathcal{S}}} & z<0.05 \mathrm{~m}-2 r_{\mathcal{S}} \\
1 & 0.05 \mathrm{~m}-2 r_{\mathcal{S}} \leq z \leq 0.05 \mathrm{~m}+2 r_{\mathcal{S}} \\
\frac{0.2 \mathrm{~m}-z}{0.15 \mathrm{~m}+2 r_{\mathcal{S}}} & z>0.05 \mathrm{~m}+2 r_{\mathcal{S}}\end{cases}
\end{aligned}
$$

such that $T_{A L E}\left(\boldsymbol{c}_{\mathcal{S}} ; r, z\right)$ is a pure translation for all $z \in\left[0.05 \mathrm{~m}-2 r_{\mathcal{S}}, 0.05 \mathrm{~m}+2 r_{\mathcal{S}}\right]$, where the reference ball is located. We make sure that the lines at $z=0.05 \mathrm{~m} \pm 2 r_{\mathcal{S}}$ are resolved by the computational mesh such that $T_{A L E}$ is differentiable within all elements. This construction of the domain map does not allow us to reduce the distance of the ball from the lower boundary to less than $r_{\mathcal{S}}$.

The reference domain $\mathcal{F}$ is basis for the finite element discretisation. In cylindrical coordinates, the ALE version of the variational formulation takes the form

$$
\begin{aligned}
m_{A L E}\left(\boldsymbol{c}_{\mathcal{S}} ; \boldsymbol{u}, \boldsymbol{v}\right) & =2 \pi \rho_{f} \int_{\mathcal{F}} r J\left(\boldsymbol{c}_{\mathcal{S}}\right) \boldsymbol{u} \cdot \boldsymbol{v} \mathrm{d} \boldsymbol{x} \\
a_{A L E}\left(\boldsymbol{c}_{\mathcal{S}} ; \boldsymbol{u}, \boldsymbol{v}\right) & =2 \pi \mu_{f} \int_{\mathcal{F}} J\left(\boldsymbol{c}_{\mathcal{S}}\right) \nabla \boldsymbol{u}\left(\begin{array}{cc}
1 & 0 \\
0 & J\left(\boldsymbol{c}_{\mathcal{S}}\right)^{-1}
\end{array}\right): \nabla \boldsymbol{v}\left(\begin{array}{cc}
1 & 0 \\
0 & J\left(\boldsymbol{c}_{\mathcal{S}}\right)^{-1}
\end{array}\right)+\frac{J\left(\boldsymbol{c}_{\mathcal{S}}\right)}{r} \boldsymbol{u}_{r} \boldsymbol{v}_{r} \mathrm{~d} \boldsymbol{x} \\
c_{A L E}\left(\boldsymbol{c}_{\mathcal{S}} ; \boldsymbol{u}, \boldsymbol{v}, \boldsymbol{w}\right) & =2 \pi \rho_{f} \int_{\mathcal{F}} r J\left(\boldsymbol{c}_{\mathcal{S}}\right) \nabla\left(\begin{array}{cc}
1 & 0 \\
0 & J\left(\boldsymbol{c}_{\mathcal{S}}\right)^{-1}
\end{array}\right)\left(\boldsymbol{u}-\partial_{t} T_{A L E}\left(\boldsymbol{c}_{\mathcal{S}}\right)\right) \cdot \boldsymbol{w} \mathrm{d} \boldsymbol{x} \\
b_{A L E}\left(\boldsymbol{c}_{\mathcal{S}} ; q, \boldsymbol{v}\right) & =-2 \pi \int_{\mathcal{F}} J\left(\boldsymbol{c}_{\mathcal{S}}\right) q\left(\boldsymbol{v}_{r}+r \partial_{r} \boldsymbol{v}_{1}+\partial_{z} \boldsymbol{v}_{2} / J\left(\boldsymbol{c}_{\mathcal{S}}\right)\right) \mathrm{d} \boldsymbol{x},
\end{aligned}
$$

where $J\left(\boldsymbol{c}_{\mathcal{S}}\right)=\operatorname{det}\left(\nabla T_{A L E}\right)$ is the determinant of the deformation gradient. 
The discretisation is realised by means of quadratic equal-order finite elements for velocity and pressure on a quadrilateral mesh of the reference domain $\mathcal{F}$, we refer to [Ric17, Section 4.4] for details on the realisation. To stabilise the inf-sup condition we use the local projection scheme as introduced by Becker and Braack [BB01], given, in ALE formulation, as

$$
s_{A L E}\left(\boldsymbol{c}_{\mathcal{S}} ; p, q\right)=\int_{\mathcal{F}} r\left(\begin{array}{cc}
\delta_{r} & 0 \\
0 & \delta_{z}
\end{array}\right) \nabla \kappa_{h}(p) \cdot\left(\begin{array}{cc}
J\left(\boldsymbol{c}_{\mathcal{S}}\right) & 0 \\
0 & J\left(\boldsymbol{c}_{\mathcal{S}}\right)^{-1}
\end{array}\right) \nabla \kappa_{h}(q)+\frac{J\left(\boldsymbol{c}_{\mathcal{S}}\right)}{r} \delta_{r} \kappa_{h}(p) \kappa_{h}(q) \mathrm{d} \boldsymbol{x} .
$$

Here $\kappa_{h}:=\mathrm{id}-i_{2 h}$ is the coarse mesh fluctuation operator that subtracts the interpolation to the mesh with double spacing and where $\delta_{r}$ and $\delta_{z}$ are local stabilisation parameters that depend on the element diameter $h$ and the time step size $k$

$$
\delta_{r}=0.1 \cdot\left(\frac{\mu_{f}}{\rho_{f} h^{2}}+\frac{1}{k}\right)^{-1}, \quad \delta_{z}=0.1 \cdot\left(\frac{\mu_{f}}{\rho_{f} h^{2} J\left(\boldsymbol{c}_{\mathcal{S}}\right)^{2}}+\frac{1}{k}\right)^{-1} .
$$

The different scaling in $r$ - and $z$-direction reflects the anisotropy induced by the ALE transformation, c.f. [BR06] or [Ric17, Section 5.3.3].

In time we discretise the Navier-Stokes equations and the rigid body problem with BDF2 time stepping in a decoupled iteration taking $10^{-8}$ as tolerance for the solid velocity and deformation update. The forces acting on the solid are evaluated by means of the Babuška-Miller Trick [BM84; Wah+19] such that quadratic finite elements let us expect fourth order convergence. Hence, each step of spatial refinement will be accompanied by two refinement of the time step.

Fluid-Rigid Body interaction in Arbitrary Lagrangian-Eulerian coordinates in three dimensions. To clarify the role of fixed body rotations of the solid and deflections of the center of mass from the $z$-axis, both of which were observed in the experimental analysis [HTR20; Hag20], we perform full three dimensional simulations. The Navier-Stokes equations are formulated in Cartesian coordinates (2.1) and the motion of the rigid body is described by

$$
\begin{aligned}
\frac{\mathrm{d}}{\mathrm{d} t} \boldsymbol{c}_{\mathcal{S}}(t)=\boldsymbol{v}_{\mathcal{S}}(t), \quad \frac{\mathrm{d}}{\mathrm{d} t} \boldsymbol{v}_{\mathcal{S}}(t) \cdot m_{\mathcal{S}} & =\left(\begin{array}{c}
0 \\
0 \\
m_{\mathcal{S}} g-\operatorname{vol}(\mathcal{S}) \rho_{f} g
\end{array}\right)+\int_{\mathcal{I}} \boldsymbol{\sigma}(\boldsymbol{u}, p) \boldsymbol{n} \mathrm{d} s, \\
\frac{\mathrm{d}}{\mathrm{d} t} \boldsymbol{\omega}_{\mathcal{S}}(t) \cdot I_{\mathcal{S}}+\boldsymbol{\omega}_{\mathcal{S}}(t) \times I_{\mathcal{S}} \boldsymbol{\omega}_{\mathcal{S}}(t) & =\int_{\mathcal{I}}\left(\boldsymbol{x}-\boldsymbol{c}_{\mathcal{S}}(t)\right) \times \boldsymbol{\sigma}(\boldsymbol{u}, p) \boldsymbol{n} \mathrm{d} s
\end{aligned}
$$

where $\boldsymbol{v}_{\mathcal{S}}(t) \in \mathbb{R}^{3}$ is the full velocity vector, $\boldsymbol{c}_{\mathcal{S}}(t) \in \mathbb{R}^{3}$ the solid's center of mass and $\boldsymbol{\omega}_{\mathcal{S}}(t) \in \mathbb{R}^{3}$ the rotational velocity vector, c.f. (2.4), (2.5), (2.6). Assuming a homogenous distribution of the density the moment of inertia is given by

$$
I_{\mathcal{S}}=\frac{8}{15} \pi \rho_{\mathcal{S}} r_{\mathcal{S}}^{5} \mathrm{Id} .
$$

It follows that the nonlinear term in the rotational ODE $(4.2)$ vanishes, since $\omega_{\mathcal{S}}(t) \times I_{\mathcal{S}} \boldsymbol{\omega}_{\mathcal{S}}(t)=\frac{8}{15} \pi \rho_{\mathcal{S}} r_{\mathcal{S}}^{5} \omega_{\mathcal{S}}(t) \times$ $\boldsymbol{\omega}_{\mathcal{S}}(t)=0$. On the surface of the sphere, the Navier-Stokes Dirichlet conditions are used to describe the velocity

$$
\boldsymbol{v}(\boldsymbol{x}, t)=\boldsymbol{v}_{\mathcal{S}}(t)+\boldsymbol{\omega}_{\mathcal{S}}(t) \times\left(\boldsymbol{x}-\boldsymbol{c}_{\mathcal{S}}\right) \text { on } \mathcal{I} .
$$

To evaluate the torque correctly through the variational formulation, i.e., by using the Babuška-Miller Trick [BM84; Wah+19], the symmetric gradient is used instead in (3.2), hence

$$
a^{3 d}(\boldsymbol{u}, \boldsymbol{v})=\mu_{f} \int_{\Omega^{3 d}}\left(\nabla \boldsymbol{u}+\nabla \boldsymbol{u}^{T}\right): \nabla \boldsymbol{v} \mathrm{d} \boldsymbol{x} .
$$

We cast the problem in standard ALE formulation and refer to [Ric17, Chapter 5] for further details. The ALE map $T_{A L E}(t)$ is chosen similar to the reduced case (4.1), but we must incorporate motion in the $x$ - $y$-plane 
and define

$$
T_{A L E}\left(\boldsymbol{c}_{\mathcal{S}} ; x, y, z\right)=\left(\begin{array}{c}
x+\boldsymbol{c}_{\mathcal{S}, 1} \cdot g_{A L E}(r(x, y)) \\
y+\boldsymbol{c}_{\mathcal{S}, 2} \cdot g_{A L E}(r(x, y)) \\
z+\left(\boldsymbol{c}_{\mathcal{S}, 3}-r_{\mathcal{S}}-0.05 \mathrm{~m}\right) f_{A L E}(z),
\end{array}\right)
$$

where $f_{A L E}(\cdot)$ is defined in $(4.1)$ while $r(\cdot, \cdot)$ and $g_{A L E}(\cdot)$ are given by

$$
r(x, y)=\max \left\{0, \min \left\{1, \frac{x-r_{\mathcal{S}}}{R-r_{\mathcal{S}}}\right\}\right\}, \quad g_{A L E}(r)=1-\frac{1}{1+\exp \left(\frac{1-2 r}{r-r^{2}}\right)}
$$

The function $r(\cdot, \cdot)$ maps the $x / y$ plane to $[0,1]$ with $r(x, y)=0$ for $\sqrt{x^{2}+y^{2}} \leq r_{\mathcal{S}}$ and $r(x, y)=1$ for $\sqrt{x^{2}+y^{2}} \geq R$. Further, $g_{A L E}$ is a smooth transition function mapping $(0,1)$ to $(0,1)$ with all derivatives being zero at 0 and 1 . On the other hand, the derivative of $f_{A L E}(z)$ is not defined at $z=0.05 \mathrm{~m} \pm 2 r_{\mathcal{S}}$. These two surfaces are however resolved by the finite element mesh to give good approximation characteristics of the ALE formulation.

Implementation. Both formulations, the reduced two dimensional ALE formulation in cylindrical coordinates and the full three dimensional ALE formulation are implemented in the finite element library Gascoigne 3D $[\mathrm{Bec}+]$. The coupling between the Navier-Stokes equation and the rigid body motion is resolved in a simple iteration until the discrepancy in velocity reached a threshold

$$
\left\|\left.\boldsymbol{v}\right|_{\mathcal{I}}-\boldsymbol{v}_{\mathcal{S}}\right\|_{\infty}<10^{-8}
$$

The nonlinearity of the Navier-Stokes equation is solved by a Newton iteration and the resulting linear systems of equations are approximated with a parallel GMRES iteration, preconditioned by a geometric multigrid solver, see [FR20]. The meshes are graded with a higher resolution close to the solid.

\subsubsection{Fluid-Rigid Body interaction in Eulerian coordinates}

As in subsubsection 4.1.1, we consider the problem as a moving domain problem for the fluid, assume the solid to be a rigid body and decouple the fluid and solid equations. For the resulting moving domain problem we use an unfitted Eulerian finite element method from [WRL20] using BDF2 time-stepping, together with Taylor-Hood elements in space, which are inf-sup stable in the CutFEM setting [GO17] with ghost-penalty stabilisation [Bur10]. In order to obtain the full convergence order of the Taylor-Hood finite element pair, we use an isoparametric mapping introduced in [Leh16] for stationary domains to realise the higher-order geometry approximation.

Transformed Nitsche terms. In the used CutFEM method, boundary conditions on unfitted boundaries are enforced using Nitsches method [Nit71]. These also need to be transformed to the rotationally symmetric setting such that we have a consistent and stable formulation.

Let $\Gamma$ be the Dirichlet boundary. Integrating the diffusion operator $a(\cdot, \cdot)$ by parts in cylinder coordinates, we find that the consistency term is

$$
n_{c}(\boldsymbol{u}, \boldsymbol{v})=-2 \pi \mu_{f} \int_{\Gamma} r(\nabla \boldsymbol{u}) \boldsymbol{n} \cdot \boldsymbol{v} \mathrm{d} S
$$

To enforce the Dirichlet boundary conditions consistently, we also scale the penalty term with $r$, such that we have

$$
n_{s}(\boldsymbol{u}, \boldsymbol{v})=2 \pi \mu_{f} \sigma \frac{k^{2}}{h} \int_{\Gamma} r \boldsymbol{u} \cdot \boldsymbol{v} \mathrm{d} S .
$$

with a penalty parameter $\sigma>0$, the velocity space's polynomial order $k$ and the local mesh diameter $h$. In total we have the consistent and symmetric Nitsche formulation of the vector valued diffusion operator 
$a_{h}(\boldsymbol{u}, \boldsymbol{v})=a(\boldsymbol{u}, \boldsymbol{v})+n_{c}(\boldsymbol{u}, \boldsymbol{v})+n_{c}(\boldsymbol{v}, \boldsymbol{u})+n_{s}(\boldsymbol{u}, \boldsymbol{v})$. Similarly we find for the pressure-coupling operator, that the Nitsche term is

$$
n_{p}(\boldsymbol{v}, q)=\int_{\Gamma} r q \boldsymbol{v} \cdot \boldsymbol{n} \mathrm{d} S
$$

so that the consistent reduced formulation is $b_{h}(\boldsymbol{v}, q)=b(\boldsymbol{v}, q)+n_{p}(\boldsymbol{v}, q)$. To implement inhomogeneous Dirichlet conditions, we then add the symmetry and penalty terms with the required boundary values to the right-hand side of the fluid equations.

Transformed ghost-penalty operators. The role of the ghost-penalty operator in the unfitted Eulerian timestepping scheme used here is twofold. As in other CutFEM discretisations, it stabilises arbitrary element cuts, such that the method is stable and the resulting matrices are well-conditioned [Bur10]. Secondly, appropriately scaled ghosty-penalties provide the necessary implicit extension for the method-of-lines approach to the discretisation of the time-derivative [LO19; BFM19; WRL20].

We use the direct-version of the ghost-penalty operator [Pre18]. Let $\mathscr{F}_{h}$ be a set of facets between neighbouring elements on which the ghost-penalty operator is to act on, c.f. [LO19; WRL20] for further details. For a facet $F \in \mathscr{F}_{h}$ such that $F=\bar{T}_{1} \cap \bar{T}_{2}$, we define the facet patch $\omega_{F}=T_{1} \cup T_{2}$. The velocity ghost-penalty operator for the rotationally symmetric formulation is then

$$
i_{h}(\boldsymbol{u}, \boldsymbol{v})=\gamma_{\boldsymbol{u}} \sum_{F \in \mathscr{F}_{h}} \int_{\omega_{F}} \frac{r}{h^{2}}\left(\boldsymbol{u}_{1}-\boldsymbol{u}_{2}\right) \cdot\left(\boldsymbol{v}_{1}-\boldsymbol{v}_{2}\right)+\frac{1}{r}\left(\boldsymbol{u}_{r, 1}-\boldsymbol{u}_{r, 2}\right)\left(\boldsymbol{v}_{r, 1}-\boldsymbol{v}_{r, 2}\right) \mathrm{d} \boldsymbol{x}
$$

where $\boldsymbol{v}_{i}=\left.\mathcal{E}^{\mathbb{P}} \boldsymbol{v}\right|_{T_{i}}$ with the canonical extension of polynomials $\mathcal{E}^{\mathbb{P}}: \mathbb{P}(T) \rightarrow \mathbb{P}\left(\mathbb{R}^{d}\right)$. The pressure ghostpenalty operator is

$$
j_{h}(p, q)=\gamma_{p} \sum_{F \in \mathscr{F}_{h}} \int_{\omega_{F}} r\left(p_{1}-p_{2}\right)\left(q_{1}-q_{2}\right) \mathrm{d} \boldsymbol{x} .
$$

With these transformed ghost-penalty operators, it is easy to show the standard ghost-penalty results in the appropriately transformed norms.

Contact algorithm. We consider a very basic contact avoidance scheme, used widely in the literature [Glo +99 ; WT06; ST08; Fre16]. The idea is to introduce an artificial (lubrication) force acting on the rigid body in the vicinity of the contact wall, which increases the closer the ball comes to the wall and acts in the direction away from the wall. This force is then added to the forces governing the motion of the rigid solid such that contact does not occur. We define this force as

$$
f_{c}(\mathcal{S})= \begin{cases}0 & \text { if } \operatorname{dist}\left(\mathcal{I}, \Gamma_{\text {bottom }}\right) \geq d i s t_{0} \\ \gamma_{c} \frac{d i s t_{0}-\operatorname{dist}\left(\mathcal{I}, \Gamma_{\text {bottom }}\right)}{\operatorname{dist}\left(\mathcal{I}, \Gamma_{\text {bottom }}\right)} & \text { if } \operatorname{dist}\left(\mathcal{I}, \Gamma_{\text {bottom }}\right)<d i s t_{0}\end{cases}
$$

where dist $_{0}$ and $\gamma_{c}$ are parameters to be chosen and $\operatorname{dist}\left(\mathcal{I}, \Gamma_{\text {bottom }}\right)$ is the minimal distance between $\mathcal{I}$ and $\Gamma_{\text {bottom. }}$. We then add this to the right-hand side of the ODE (2.4) and carry this thought, so that the right-hand side of $(2.7)$ becomes $\frac{\rho_{\mathcal{S}}-\rho_{\mathcal{F}}}{\rho_{\mathcal{S}}} g+\frac{\boldsymbol{F}_{y}+f_{c}}{\operatorname{vol}(\mathcal{S}) \rho_{\mathcal{S}}}$.

Implementation. This discretisation is implemented using the finite-element library netgen/NGSolve, see [Sch97; Sch14] and ngsolve.org, together with the add-on package ngsxfem [xfem1.3] for unfitted finite element functionality.

The background mesh is constructed by defining a local mesh parameter $h_{\text {inner }}$ on the left of the reduced domain where $r<2 d_{\mathcal{S}} / 3$ and then creating a shape regular mesh with $h=h_{\max }$ in the remainder of the domain. This is to obtain more accurate boundary integrals, i.e., when computing the forces acting on the ball. In the Rubber22 setting we choose $h_{\text {inner }}=h_{\max } / 4$ and in the PTFE6 case as $h_{\text {inner }}=h_{\max } / 7$. This is to obtain background meshes with a similar number of elements in each settings. On the active part of the mesh we consider standard $\mathbb{P}^{2} / \mathbb{P}^{1}$ Taylor-Hood elements. 
The Nitsche parameter is taken as $\sigma=100$, the extension ghost-penalty parameter is $\gamma_{\boldsymbol{u}, e}=0.1$ and the cut-stability ghost-penalty parameter $\gamma_{\boldsymbol{u}, s}=\gamma_{p, s}=0.01$ and the extension strip width parameter is $c_{\delta}=4$. See [WRL20] for details on these parameters.

The contact parameters are tuned with respect to the PTFE6 jump height, since the model cannot be expected to resolve the elastic nature of rubber. For the PTFE6 we take the contact model parameters dist $_{0}=2 \cdot 10^{-5}$ and $\gamma_{c}=0.38$. Since the mass of the Rubber 22 ball as approximately 31.6 times larger than the PTFE6 ball, we take contact model parameters which are appropriately larger, such that the resulting acceleration acting on the balls is comparable. For the Rubber22 computations we take $d_{i s t}=2 \cdot 10^{-5}$ and $\gamma_{c}=12$.

Each time-step is iterated between the fluid system and the solid ODE until the system is solved. We consider the system as solved, when the update of the balls velocity in an iteration is less than $10^{-8}$.

\subsubsection{Fluid-Structure interaction in fully Eulerian coordinates}

We consider the full fluid-structure interaction problem including contact with $\Gamma_{\text {bottom }}$. We adopt here a fully Eulerian approach for the FSI system in order to enable the transition to contact [Dun06; Ric13; Fre16]. To allow for an implicit inclusion of the contact conditions into the system (see below), we use a Nitsche-based method for the FSI coupling as presented in [BFF20].

Solid bilinear form and FSI coupling. The fluid bilinear form has already been detailed in (3.4). To introduce the solid form, we denote the reduced solid domain by $\mathcal{S}(t)$ and define the bilinear form corresponding to the linear elasticity equations in Eulerian coordinates (2.2) by

$$
A_{s}(\boldsymbol{d}, \dot{\boldsymbol{d}} ; \boldsymbol{w}, \boldsymbol{z}):=m_{s}\left(\partial_{t} \dot{\boldsymbol{d}}-\dot{\boldsymbol{d}} \cdot \nabla \dot{\boldsymbol{d}}, \boldsymbol{w}\right)+a_{s}(\boldsymbol{d}, \boldsymbol{w})+m_{\dot{\boldsymbol{d}}}\left(\partial_{t} \boldsymbol{d}-\dot{\boldsymbol{d}} \cdot \nabla \boldsymbol{d}+\dot{\boldsymbol{d}}, \boldsymbol{z}\right),
$$

where

$$
\begin{gathered}
m_{s}(\boldsymbol{d}, \boldsymbol{w}):=2 \pi \rho_{s} \int_{\mathcal{S}} r \boldsymbol{d} \cdot \boldsymbol{w} \mathrm{d} \boldsymbol{x}, \quad a_{s}(\boldsymbol{d}, \boldsymbol{w}):=2 \pi \int_{\mathcal{S}} r \nabla \sigma_{s}(d): \nabla \boldsymbol{w}+\sigma_{s, r} \boldsymbol{w}_{r} \mathrm{~d} \boldsymbol{x}, \\
m_{\dot{d}}(\boldsymbol{d}, \boldsymbol{w}):=2 \pi \int_{\mathcal{S}} \boldsymbol{d} \cdot \boldsymbol{w} \mathrm{d} \boldsymbol{x} .
\end{gathered}
$$

and

$$
\sigma_{s}=2 \mu_{s} E(\boldsymbol{d})+\lambda_{s}\left(\operatorname{tr}(E(\boldsymbol{d}))+\frac{1}{r} \boldsymbol{d}_{r}\right) I, \quad \sigma_{s, r}=\frac{2 \mu_{s}+\lambda_{s}}{r} \boldsymbol{d}_{r}+\lambda_{s} \operatorname{tr}(E(\boldsymbol{d})) .
$$

Moreover, we make use of the Nitsche terms defined in subsubsection 4.1.2 to impose the FSI coupling conditions $(2.3)$

$$
n(\boldsymbol{u}, p, \dot{\boldsymbol{d}} ; \boldsymbol{v}, q, \boldsymbol{w}):=n_{s}(\boldsymbol{u}-\dot{\boldsymbol{d}}, \boldsymbol{v}-\boldsymbol{w})+n_{c}(\boldsymbol{u}, \boldsymbol{v}-\boldsymbol{w})+n_{c}(\boldsymbol{v}, \boldsymbol{u}-\dot{\boldsymbol{d}})+n_{p}(\boldsymbol{v}-\boldsymbol{w}, p)-n_{p}(\boldsymbol{u}-\dot{d}, q) .
$$

Note the negative sign in front of the last term, which is required to ensure stability of the FSI formulation, see [BF14].

Discretisation and stabilisation. In order to resolve the interface $\mathcal{I}$ within the discretisation, we use the locally modified finite element method introduced in [FR14]. This fitted finite element method is based on a coarse unfitted patch mesh, which is independent of the interface location. The coarse cells are then divided in such a way into sub-triangles and sub-quadrilaterals that the interface is resolved in a linear approximation. In this work, we use equal-order locally modified finite elements of first order, in combination with an anisotropic edge-oriented pressure stabilisation term $s_{p}(p, q)$, see [Fre19] for the details. We denote the locally modified finite element space of first order by $X_{h}^{1}$. The discrete spaces for fluid velocity and pressure $\boldsymbol{V}_{h}$ and $Q_{h}$ and for solid displacement and velocity $\boldsymbol{W}_{h}$ and $Z_{h}$ are given by applying the respective 
Dirichlet conditions to the locally modified finite element space $X_{h}^{1}$ and by restricting the degrees of freedom to the fluid or solid sub-domain, respectively.

In addition, we add a stabilisation term $s_{\boldsymbol{d}}(\dot{d}, z)$ of artificial diffusion type to the solid equations, see [Fre16], and a consistent stabilisation at the boundary $\Gamma_{\mathrm{sym}}$ corresponding to the rotational axis to ensure that the second boundary condition (3.3) is accurately imposed in the discrete formulation

$$
s_{\boldsymbol{d}}(\dot{d}, \boldsymbol{z})=\alpha_{d} h^{2}(r \nabla \dot{d}, \nabla \boldsymbol{z})_{\mathcal{S}}, \quad s_{r}(\boldsymbol{u}, \boldsymbol{v}):=\alpha_{\mathrm{sym}} \rho_{f} \mu_{f} \int_{\Gamma_{\mathrm{sym}}} h_{n}^{2} h_{\tau} \partial_{r} \boldsymbol{u}_{z} \partial_{r} \mathrm{~d} S .
$$

Here, $h_{n}$ and $h_{\tau}$ refer to the cell-sizes in normal and tangential direction, respectively. We summarise the stabilisation terms in the bilinear form

$$
s(\boldsymbol{u}, p, \dot{d} ; \boldsymbol{v}, q, \boldsymbol{z})=s_{p}(p, q)+s_{\boldsymbol{d}}(\dot{d}, \boldsymbol{z})+s_{r}(\boldsymbol{u}, \boldsymbol{v}) .
$$

For time discretisation, we use the modified $\mathrm{dG}(0)$ time discretisation presented in [FR17], which can be seen as a variant of the $\mathrm{dG}(0)$ /backward Euler methods that considers the movement of the interface in each space-time slab. The interface positions and the domain affiliations are updated explicitly based on the displacement $d\left(t_{n-1}\right)$ of the previous time-step and using the Initial Point Set/Backwards Characteristics method [Dun06; Fre16]. This means that we set

$$
\mathcal{I}^{n}:=\mathcal{I}\left(d\left(t_{n-1}\right)\right), \quad \mathcal{S}^{n}:=\mathcal{S}\left(d\left(t_{n-1}\right)\right), \quad \mathcal{F}^{n}:=\mathcal{F}\left(d\left(t_{n-1}\right)\right) .
$$

Contact treatment. When a part $\mathcal{I}_{c}:=\mathcal{I} \cap \Gamma_{\text {bottom }}$ of $\mathcal{I}$ enters into contact with $\Gamma_{\text {bottom }}$, the FSI conditions need to be substituted with appropriate contact conditions. It has been noted in [BFF20] and [Age+19] that although the fluid layer between ball and lower wall vanishes (from a macroscopical perspective), an extension of the fluid forces to the contact surface $\mathcal{I}_{c}$ has to be considered to obtain a physically relevant contact formulation. Here we use the simplest possible numerical approach, which is to relax the no-penetration condition by a small $\epsilon=\epsilon(h)>0$, such that a very thin mesh-dependent fluid layer remains at all times.

The distance to $\Gamma_{\text {bottom }}$ depends on the (Eulerian) displacement $\boldsymbol{d}\left(t_{n}\right)$ in the following way

$$
\operatorname{dist}\left(\mathcal{I}\left(t_{n}\right), \Gamma_{\text {bottom }}\right)=\operatorname{dist}\left(\mathcal{I}\left(t_{n-1}\right), \Gamma_{\text {bottom }}\right)+\boldsymbol{d}_{n}\left(t_{n}\right)-\boldsymbol{d}_{n}\left(t_{n-1}\right) .
$$

The contact conditions, relaxed by a small $\epsilon=\epsilon(h)>0$, read

$$
\operatorname{dist}\left(\mathcal{I}\left(t_{n}\right), \Gamma_{\text {bottom }}\right) \leq \epsilon, \quad \llbracket \sigma_{n} \rrbracket \leq 0, \quad \llbracket \sigma_{n} \rrbracket\left(\operatorname{dist}\left(\mathcal{I}\left(t_{n}\right), \Gamma_{\text {bottom }}\right)-\epsilon\right)=0 \quad \text { on } \mathcal{I}^{n}=\mathcal{I}\left(t_{n-1}\right),
$$

where

$$
\llbracket \sigma_{n} \rrbracket:=n^{T} \sigma_{s} n-n^{T} \sigma_{f} n .
$$

In other words the relaxation means that the contact conditions are already applied at an $\epsilon$-distance from $\Gamma_{\text {bottom }}$. The three conditions (4.3) can be equivalently formulated in equality form with an arbitrary $\gamma_{C}>$ $0[\mathrm{AC} 91]$

$$
\llbracket \sigma_{n} \rrbracket=-\gamma_{C}[\underbrace{\operatorname{dist}\left(\mathcal{I}\left(t_{n}\right), \Gamma_{\text {bottom }}\right)-\epsilon-\gamma_{C}^{-1} \llbracket \sigma_{n} \rrbracket}_{=: P_{\gamma}\left(\boldsymbol{d}, \llbracket \sigma_{n} \rrbracket\right)}]_{+} \quad \text { on } \mathcal{I}^{n},
$$

where $[\cdot]_{+}$stands for the positive part of $(\cdot)$. The contact parameter $\gamma_{C}$ will be chosen as $\gamma_{C}=\gamma_{C}^{0} \lambda_{s} h^{-1}$, the relaxation parameter as $\epsilon=\epsilon_{0} h_{y}$, where $h_{y}$ denotes the cell size in vertical direction at the bottom of the cylinder.

Note that (4.4) includes both the FSI coupling and the contact condition, as in absence of contact, it is exactly the FSI interface condition in normal direction. For this reason the transition between FSI coupling and contact conditions can be included easily in a fully implicit fashion in the variational formulation.

The final variational formulation reads:

Find $(\boldsymbol{u}, p, \boldsymbol{d}, \dot{\boldsymbol{d}}) \in \boldsymbol{V}_{h} \times Q_{h} \times \boldsymbol{W}_{h} \times \boldsymbol{Z}_{h}$, such that for all $(\boldsymbol{v}, q, \boldsymbol{w}, \boldsymbol{z}) \in \boldsymbol{V}_{h} \times Q_{h} \times \boldsymbol{W}_{h} \times \boldsymbol{Z}_{h}$ it holds

$$
\begin{aligned}
A_{f}(\boldsymbol{u}, p ; \boldsymbol{v}, q)+A_{s}(\boldsymbol{d}, \dot{\boldsymbol{d}} ; \boldsymbol{w}, \boldsymbol{z})+n(\boldsymbol{u}, p, \dot{\boldsymbol{d}} ; \boldsymbol{v}, q, \boldsymbol{w}) & +s(\boldsymbol{u}, p, \dot{d} ; \boldsymbol{v}, q, \boldsymbol{z}) \\
& +\left(r\left[P_{\gamma}\left(\boldsymbol{d}, \llbracket \sigma_{n} \rrbracket\right)\right]_{+}, \boldsymbol{w}_{n}\right)_{\mathcal{I}}=\left(r \boldsymbol{f}_{s}, \boldsymbol{w}\right)_{\Omega} .
\end{aligned}
$$




\begin{tabular}{|c|c|c|c|c|c|c|c|c|c|c|}
\hline \multicolumn{5}{|c|}{ Discretisation } & \multicolumn{6}{|c|}{ Results } \\
\hline Method & {$\left[h_{\min }, h_{\max }\right]$} & $\Delta t$ & dof & nze & $t_{*}$ & $v_{*}$ & $f_{*}$ & $t_{\text {cont }}$ & $t_{\text {jump }}$ & $d_{\text {jump }}$ \\
\hline \multirow[t]{5}{*}{ ALE } & {$[0.00008,0.004]$} & $1 / 200$ & 7.54 & 0.34 & 0.542134 & -0.3122077 & $1.11393 \cdot 10^{-3}$ & - & - & - \\
\hline & {$[0.00004,0.002]$} & $1 / 800$ & 23.88 & 1.10 & 0.539231 & -0.3139245 & $1.11982 \cdot 10^{-3}$ & - & - & - \\
\hline & {$[0.00002,0.001]$} & $1 / 3200$ & 83.07 & 3.90 & 0.539026 & -0.3139931 & $1.12008 \cdot 10^{-3}$ & - & - & - \\
\hline & & & \multirow{2}{*}{\multicolumn{2}{|c|}{$\begin{array}{l}\text { extrapolate } \\
\text { order (in } h \text { ) }\end{array}$}} & 0.539010 & -0.3139960 & $1.12021 \cdot 10^{-3}$ & & & \\
\hline & & & & & 3.8 & 4.6 & 4.0 & & & \\
\hline \multirow[t]{3}{*}{$\mathrm{CutFEM}^{4}$} & {$[0.00114,0.008]$} & $1 / 2000$ & & & 0.579181 & -0.2884462 & $1.11897 \cdot 10^{-3}$ & 0.600328 & 0.017827 & $1.33974 \cdot 10^{-3}$ \\
\hline & {$[0.00057,0.004]$} & $1 / 2000$ & & & 0.536698 & -0.3164086 & $1.12723 \cdot 10^{-3}$ & 0.556037 & 0.030627 & $2.70681 \cdot 10^{-3}$ \\
\hline & {$[0.00029,0.002]$} & $1 / 2000$ & & & 0.530537 & -0.3213762 & $1.12122 \cdot 10^{-3}$ & 0.549543 & 0.030749 & $2.69250 \cdot 10^{-3}$ \\
\hline \multirow[t]{2}{*}{ FSI } & {$[0.00032,0.00562]$} & {$[1 / 128000,1 / 1000]$} & 59.5 & 1.24 & 0.539918 & -0.3147132 & - & 0.558503 & 0.027515 & $1.81716 \cdot 10^{-3}$ \\
\hline & {$[0.00016,0.00281]$} & {$[1 / 128000,1 / 1000]$} & 236.6 & 5.40 & 0.535625 & -0.3159600 & - & 0.554358 & 0.028015 & $2.06163 \cdot 10^{-3}$ \\
\hline Experiment & & & & & 0.516403 & -0.330987 & - & 0.534503 & 0.02792 & $2.21170 \cdot 10^{-3}$ \\
\hline
\end{tabular}

Table 4.: Results for the PTFE6 set-up. ${ }^{5}$

Implementation. The described algorithms and equations have been implemented in the finite element library Gascoigne3d $[\mathrm{Bec}+]$. We use a Cartesian finite element mesh, which is highly refined in the region where contact takes place. We start with a relatively coarse time-step (e.g., $\Delta t=2 \cdot 10^{-3}$ ), which is reduced sequentially, when the ball gets close to the bottom.

We use the following numerical parameters

$$
\gamma_{C}^{0}=1, \quad \sigma=10^{5}, \quad \alpha_{d}=1, \quad \alpha_{\mathrm{sym}}=10^{3} .
$$

The contact relaxation parameter is chosen $\epsilon_{0}=\frac{1}{8}$ for the Rubber22 test case and $\epsilon_{0}=\frac{1}{4}$ for PTFE6.

\subsection{Results}

In the following we shall abbreviate the methods described in subsubsection 4.1.1 as $A L E$, in subsubsection 4.1.2 as CutFEM and in subsubsection 4.1.3 as FSI.

\subsubsection{PTFE6}

Our quantitative results for the PTFE6 set-up are presented in Table 4. Figure 3 shows the distance between the bottom of the ball to the bottom of the fluid domain over time from the experimental data and the all three numerical methods.

If we look at the pre-contact quantities of interest in Table 4 before, we see that all methods give very similar results for the given quantities. Looking at the velocity of the PTFE6 ball, we see that the numerical values are within a relative error of $5.1 \%$ of the experiment on the finest discretisations. Taking into account, that these results ignore the $2.0 \mathrm{~mm}$ deflection from the $z$-axis observed in the experimental data, we consider this to be acceptable. Since the elastic effects of the particle appear to be negligible in this phase of the problem and due to the known good approximation properties of the ALE method, we consider these to be the most accurate values for future comparison.

Looking at the quantities of interest in the later phase, we see that in both the CutFEM and FSI methods, contact occurs later than in the experiment. This is consistent with the smaller speed of the particle compared to the experiments as observed above. With respect to the jump, we see that both methods capture the rebound dynamics, since both the point in time at which the peak of the rebound is realised and the size of the jump are consistent with the experiment. As the CutFEM contact parameters were tuned with respect to the size of this jump, this is unsurprising. However, since the contact force only acts for a very small number of time-steps, the fact that the time at which the rebound is maximal is also captured well, shows that even after contact, the system is still approximated well by the fluid-rigid body system. Nevertheless, it is clear that the FSI system captures the dynamics much more accurately and without the need of essentially unknowable parameters in the model. 


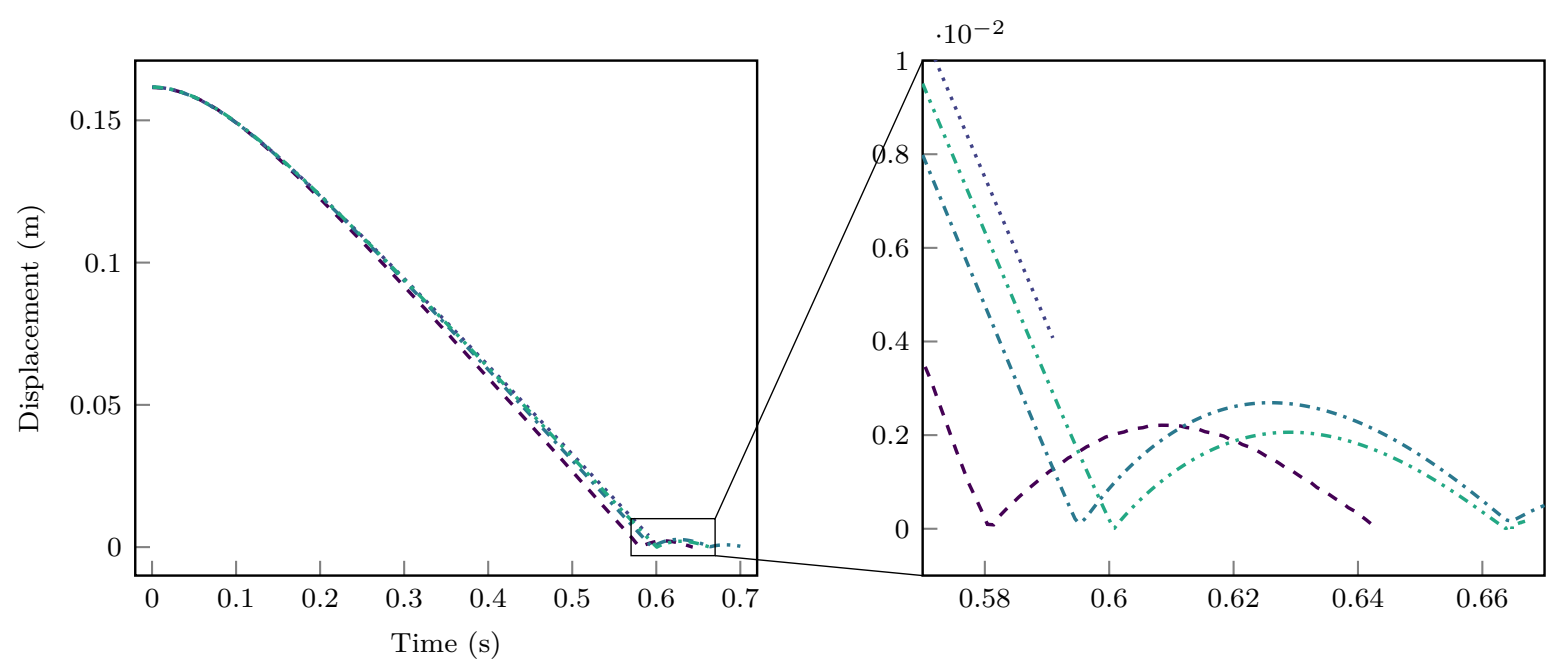

\begin{tabular}{|c|c|}
\hline $\begin{array}{l}\text { - - } \quad \text { Experimental data [Hag20] } \\
\text { - - - CutFEM: } h \in[0.00029,0.002], \Delta t=1 / 2000\end{array}$ & $\begin{array}{l}h \in[0.00002,0.001], \Delta t=1 / 3200 \\
.00016,0.00281], \Delta t=[1 / 128000,1 / 1000]\end{array}$ \\
\hline
\end{tabular}

Figure 3.: The distance between the bottom of the ball and the bottom of the tank: Experimental and numerical results for the PFTE6 set-up.

\subsubsection{Rubber22}

Our results for the Rubber22 problem are presented in Table 5. The height of the ball over time can be seen in Figure 4. To illustrate the applicability of our spatially reduced formulation, we solve the fluid-rigid body system in full three spatial dimensions using the ALE approach. These results are also given in Table 5, while a visualisation of the velocity and pressure solution at $t=0.45 \mathrm{~s}$ from the three dimensional ALE computation can be seen in Figure 5 .

We again start by inspecting the pre-contact results in Table 5 . We observe that $t_{*}, v_{*}$ and $f_{*}$ are very similar for all methods. However, here the discrepancy between the numeric and experimental velocity value is significantly smaller, with the relative difference being between $2.1 \%$ and $0.6 \%$ on the finest discretisations. We note at this point, that the deviation from the $z$-axis in this experiment was less than $7.5 \cdot 10^{-1} \mathrm{~mm}$. This shows that even for a more elastic material, a fluid-rigid body system can capture the pre-contact dynamics as well as a full FSI model. Furthermore, we see that with perfect initial data and spatial symmetry, our spatially reduced model, derived under the assumption of a rotationally symmetric solution, captures the dynamics as well as the significantly more computationally expensive full three dimensional computation.

Looking at the numbers for the contact and rebound dynamics, we again see that the time of contact is similar for both the CutFEM and FSI methods and this matches the experimental time with an error of less than 5\%. For the CutFEM method we clearly see that the PTFE tuned parameters are not able to capture the rebound dynamics well. In fact, the size of the rebound is approximately 3 times larger than the physical rebound. This shows that the use of an artificial lubrication force, as considered in a variety of other literature, can lead to physically meaningful results but is heavily dependent on the "correct" choice of parameters for which there is no a priori knowledge. For the FSI model, we see that the overall dynamics are captured well. We also observe that - while the pre-contact dynamics are essentially independent of the elasticity parameters - a variation of the elasticity modulus $E_{s}$ changes the rebound height $d_{\text {jump }}$ significantly. Here, a softer material $\left(E_{s}=2 \cdot 10^{6} \mathrm{~Pa}\right)$ leads to a larger rebound, as more elastic energy is taken up through the deformation during the impact.

\footnotetext{
${ }^{4}$ The values of dof and nze is different in every time step, as the active level set domain changes on a constant mesh. We therefore take rounded values after the first assembly of the system. This is reasonable, since the mesh is quasi uniform.

${ }^{5}$ dof: Unconstrained degrees of freedom in thousands, nze: non-zero entries of the (linearised) system in millions.
} 


\begin{tabular}{|c|c|c|c|c|c|c|c|c|c|c|}
\hline \multicolumn{5}{|c|}{ Discretisation } & \multicolumn{6}{|c|}{ Results } \\
\hline Method & {$\left[h_{\min }, h_{\max }\right]$} & $\Delta t$ & dof & nze & $t_{*}$ & $v_{*}$ & $f_{*}$ & $t_{\text {cont }}$ & $t_{\text {jump }}$ & $d_{\text {jump }}$ \\
\hline \multirow[t]{5}{*}{ ALE } & {$[0.0004,0.004]$} & $1 / 200$ & 6.9 & 0.31 & 0.4559045 & -0.303002 & $1.13262 \cdot 10^{-2}$ & - & - & - \\
\hline & {$[0.0002,0.002]$} & $1 / 800$ & 21.5 & 0.99 & 0.4553551 & -0.303552 & $1.13107 \cdot 10^{-2}$ & - & - & - \\
\hline & {$[0.0001,0.001]$} & $1 / 3200$ & 73.8 & 3.45 & 0.4553334 & -0.303616 & $1.13117 \cdot 10^{-2}$ & - & - & - \\
\hline & & & \multirow{2}{*}{\multicolumn{2}{|c|}{$\begin{array}{l}\text { extrapolate } \\
\text { order (in } h \text { ) }\end{array}$}} & 0.4553325 & -0.303625 & - & - & - & - \\
\hline & & & & & 4.6 & 3.1 & - & - & - & - \\
\hline \multirow[t]{3}{*}{ ALE 3D } & {$[0.0008,0.032]$} & $1 / 200$ & 18.43 & 4.05 & 0.453695 & -0.304252 & $1.14252 \cdot 10^{-2}$ & - & - & - \\
\hline & {$[0.0004,0.016]$} & $1 / 800$ & 68.52 & 15.37 & 0.456145 & -0.302170 & $1.14493 \cdot 10^{-2}$ & - & - & - \\
\hline & {$[0.0002,0.008]$} & $1 / 3200$ & 304.4 & 69.33 & 0.455929 & -0.302667 & $1.13557 \cdot 10^{-2}$ & - & - & - \\
\hline \multirow[t]{3}{*}{$\mathrm{CutFEM}^{4}$} & {$[0.00200,0.008]$} & $1 / 2000$ & & & 0.453455 & -0.3095423 & $1.09094 \cdot 10^{-2}$ & 0.524979 & 0.098086 & $7.30815 \cdot 10^{-3}$ \\
\hline & {$[0.00100,0.004]$} & $1 / 2000$ & & & 0.454081 & -0.3088412 & $1.11082 \cdot 10^{-2}$ & 0.525502 & 0.110672 & $1.14772 \cdot 10^{-2}$ \\
\hline & {$[0.00050,0.002]$} & $1 / 2000$ & & & 0.453789 & -0.3062202 & $1.12922 \cdot 10^{-2}$ & 0.526010 & 0.121606 & $1.36874 \cdot 10^{-2}$ \\
\hline \multirow[t]{2}{*}{ FSI $\left(E_{s}=5 \cdot 10^{6}\right)$} & {$[0.0010,0.004]$} & {$[1 / 2000,1 / 500]$} & 51.4 & 1.15 & 0.446020 & -0.3206889 & - & 0.515197 & 0.07914 & $2.68298 \cdot 10^{-3}$ \\
\hline & {$[0.0005,0.002]$} & {$[1 / 2000,1 / 500]$} & 204.6 & 4.72 & 0.449821 & -0.3113851 & - & 0.521487 & 0.083323 & $3.71986 \cdot 10^{-3}$ \\
\hline \multirow{2}{*}{ FSI $\left(E_{s}=2 \cdot 10^{6}\right)$} & {$[0.0010,0.004]$} & {$[1 / 2000,1 / 500]$} & 51.4 & 1.15 & 0.446012 & -0.3207151 & - & 0.516197 & 0.0925 & $5.29247 \cdot 10^{-3}$ \\
\hline & {$[0.0005,0.002]$} & {$[1 / 2000,1 / 500]$} & 204.6 & 4.72 & 0.449827 & -0.3113759 & - & 0.522087 & 0.0905 & $5.50146 \cdot 10^{-3}$ \\
\hline Experiment & & & & & 0.469137 & -0.309301 & - & 0.544021 & 0.089492 & $4.41485 \cdot 10^{-3}$ \\
\hline
\end{tabular}

Table 5.: Results for the Rubber22 set-up. ${ }^{5}$

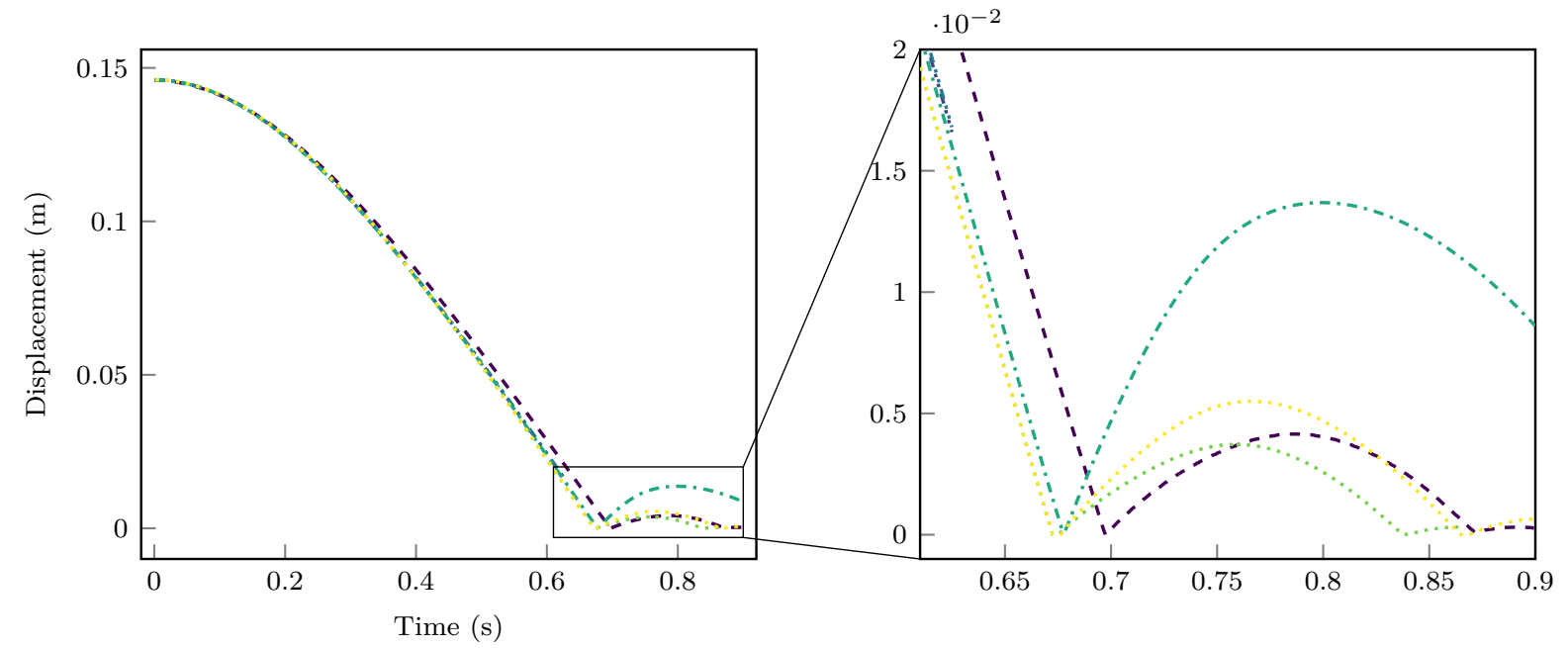

\begin{tabular}{|cccc|}
\hline$\cdots-$ & Experimental data $[$ Hag20] & $\cdots \cdots$ & ALE: $h \in[0.0001,0.001], \Delta t=1 / 3200$ \\
$\cdots \cdots$ & ALE 3D: $h \in[0.0002,0.008], \Delta t=1 / 3200$ & $\cdots$ & CutFEM: $h \in[0.0005,0.002], \Delta t=1 / 2000$ \\
$\cdots$ & FSI: $\left(E_{s}=5 \cdot 10^{6}\right)$ & FSI: $\left(E_{s}=2 \cdot 10^{6}\right)$ \\
& $h \in[0.00047,0.00196], \Delta t=[1 / 2000,1 / 500]$ & $h \in[0.00047,0.00196], \Delta t=[1 / 2000,1 / 500]$ \\
\hline
\end{tabular}

Figure 4.: The distance between the bottom of the ball and the bottom of the tank: Experimental and numerical results for the Rubber22 set-up. 

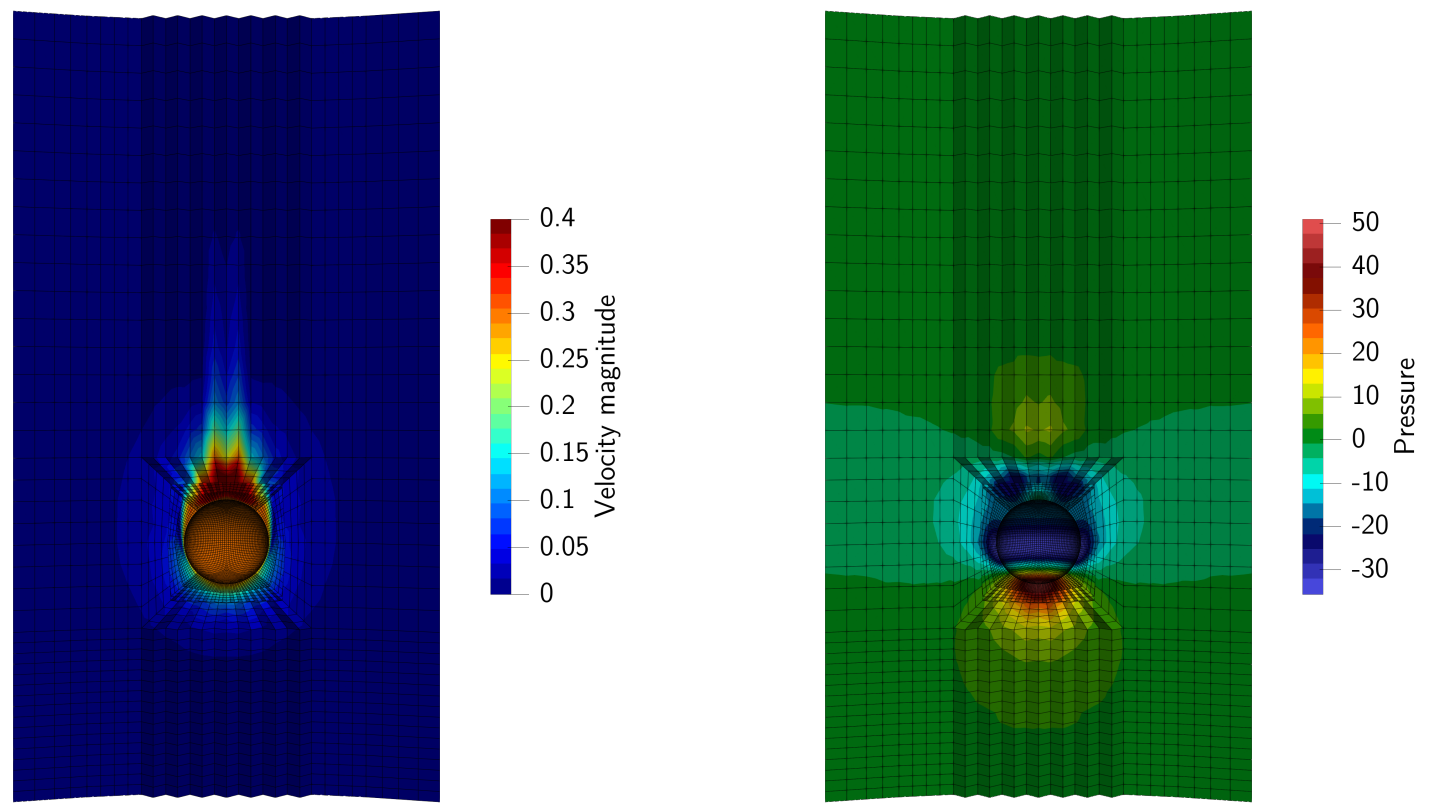

Figure 5.: Velocity and pressure solution at $t=0.45 \mathrm{~s}$ for the Rubber22 case, resulting from the ALE 3D computation with $h_{\max }=0.016$ and $\Delta t=0.005 \mathrm{~s}$.

\subsubsection{Three dimensional computation including rotational effects}

One of the challenges in performing the experimental study [HTR20] was to limit the horizontal deflection of the falling objects, i.e., to keep them close to the center line. Identifying the source of these three dimensional effects is one of the intriguing questions for future research. The cause may be found in a complex solution pattern of the Navier-Stokes equations, in material inaccuracies like non-uniform distribution of the mass or the surface roughness but also in experimental inaccuracies, e.g. during the release process.

The three dimensional simulations based on the ALE formulation presented above in subsubsection 4.2.2 did not show any three dimensional effect if the configuration is fully symmetric and the ball is released at the center line, e.g. $\boldsymbol{c}_{\mathcal{S}}=(0,0, d(0))$. To investigate the stability of the Navier-Stokes rigid body system we consider further numerical simulations based on the Rubber22 case with distorted initial values. We start the simulation with the initial data

$$
\begin{aligned}
\boldsymbol{c}_{\mathcal{S}} & =\left(10^{-3} \chi_{x}, 10^{-3} \chi_{y}, 0.1461203\right) \mathrm{m}, \\
\boldsymbol{v}_{\mathcal{S}}(0) & =\left(4 \cdot 10^{-3} \chi_{x}, 4 \cdot 10^{-3} \chi_{y}, 0\right) \mathrm{m} \mathrm{s}^{-1} \\
\boldsymbol{\omega}_{\mathcal{S}}(0) & =\left(\chi_{1}, \chi_{2}, \chi_{2}\right) 2 \pi \cdot 10^{-2} \mathrm{~s}^{-1},
\end{aligned}
$$

where $\chi_{x}, \chi_{y}, \chi_{1}, \chi_{2}, \chi_{3} \stackrel{\text { iid }}{\sim} \mathcal{N}(0,1)$ are normally distributed random numbers with mean zero and standard deviation 1.

Figure 6 shows the results for multiple experiments based on randomly chosen initial data. The left plot shows the projection of the centre of mass onto the $x-y$ plane. These results show that numerical simulations cannot predict a substantial deflection from the center line, if an initial deflection is prescribed. While the rigid solids are indeed further removed from the center, the effect is small and the objects remain within $3 \mathrm{~mm}$ of the center. On the right, we show the velocity of the particles. The upper figure shows the horizontal velocity component while the lower plot gives the dominant vertical velocity. Here, we indeed see a substantial impact of the initial disturbances. When the solid comes close to the lower boundary, a deflection to the sides gets visible. We note that these ALE simulations crash before contact is established. At final time 

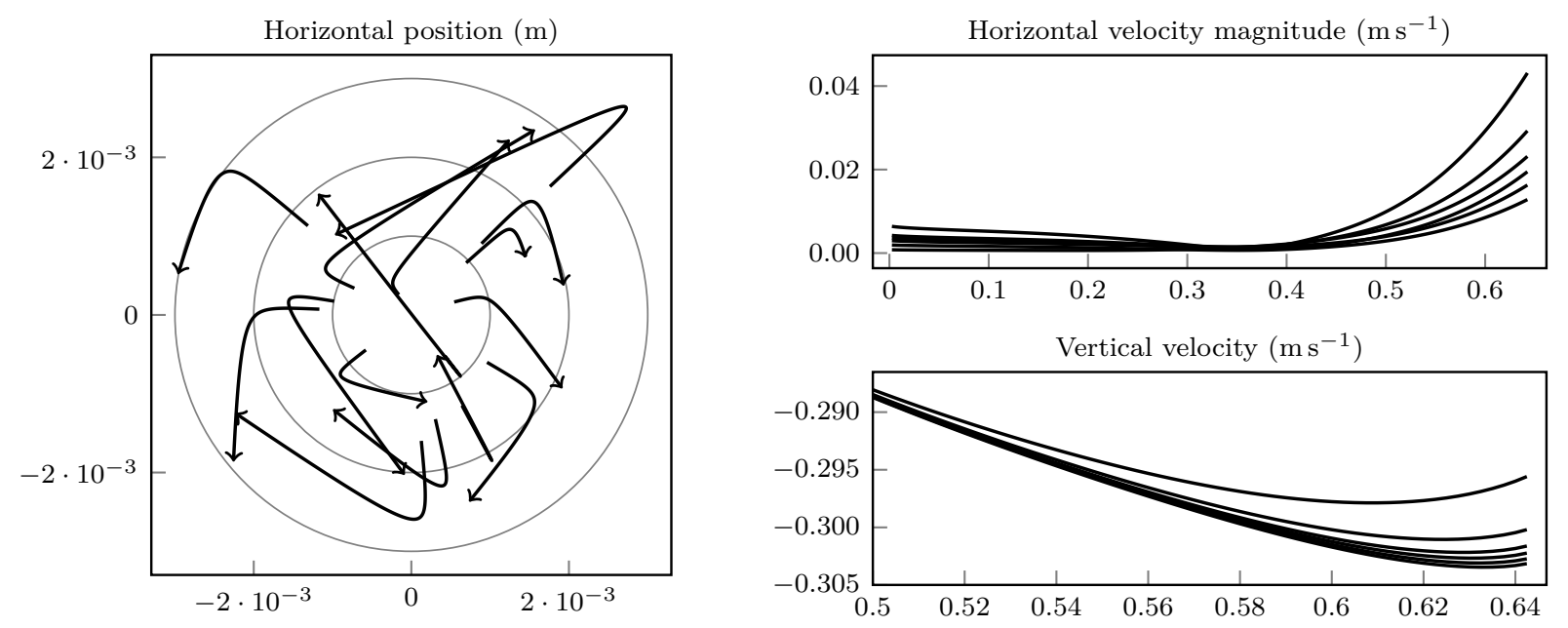

Figure 6.: Left: View from below. Deflection of the center of mass from the centreline $(0,0)$ for 15 experiments starting with random initial deviations each. Right: velocity of the particles for 6 experiments. The upper figure shows the horizontal velocity component $\sqrt{\boldsymbol{v}_{\mathcal{S}, 1}^{2}+\boldsymbol{v}_{\mathcal{S}, 2}^{2}}$ and the lower plot gives the vertical velocity close to the bottom for $t \geq 0.5 \mathrm{~s}$.

$t \approx 0.64 \mathrm{~s}$ this distance between the lower boundary is still slightly larger than one radius $r_{\mathcal{S}}=0.011 \mathrm{~m}$. Since the horizontal velocity is beginning to increase significantly here, further simulations, in which the particle is able to get closer to the bottom boundary, are of interest. We deduce from these results, that small fluctuations during the release process can indeed explain the small horizontal displacement observed during the experiments for the PTFE and Rubber particles and the discrepancy between the experimental and numerical realisations of our quantities of interest. However, these computations also show, that very large horizontal displacements, as observed in [HTR20; Hag20] for example for the POM particle, cannot be solely explained through this.

\section{Summary \& Conclusion}

We have presented two set-ups for a fluid structure interaction problem with solid contact, based on the physical experiments described in [HTR20] and the data available at [Hag20]. We computed these set-ups using a spatially reduced model under the assumption of rotational symmetry in cylinder coordinates. For the discretisation, we used a fitted ALE and unfitted CutFEM approach within a fluid-rigid body model and a fully Eulerian FSI approach in a fluid-elastic structure model capable of resolving solid contact.

We showed how each of these discretisations is able to capture the pre-contact dynamics observed in the physical experiment within a margin of $5.1 \%$ to $0.6 \%$, even though this ignored any horizontal motion observed in the experimental data. Using a full three dimensional ALE discretisation, we saw that the spatially reduced approach did indeed result in meaningful results under the assumption of perfect initial conditions at a fraction of the computational cost. Furthermore, we presented computation with disturbed initial data. From these results we deduced, that the observed horizontal motion in the PTFE and Rubber experiments is within the scope explainable by imperfect starting conditions. This shows that a fluid-rigid body model is suitable for this type of problem before solid contact occurs.

With respect to the contact dynamics, we were able to show that the Eulerian FSI discretisation with contact treatment is able to reproduce the spatial and temporal dynamics observed in the experiments very well. This is even though the theoretical modelling of such contact dynamics is not yet fully understood. The moving domain CutFEM approach together with the artificial contact treatment showed that this type of contact treatment can result in physically meaningful results when the artificial parameters are chosen "correctly" and the extent to which the artificial parameters are material dependent. 
The resulting data sets, the source code for the fluid-rigid body discretisations as well as some simplified examples are available in the zenodo repository [Wah+20].

We conclude that the two discussed set-ups are well suited for the validation of fluid structure interaction models in the moderate Reynolds number fluid regime both before and after contact. To the best of our knowledge this is the first example of a computational FSI set-up with rebound contact dynamics which is validated by experimental data. We note that the PTFE6 set-up is better suited for validation of contact and rebound models, since the model parameters are known precisely. On the other hand, the Rubber22 scenario is well suited to validate models before or without contact, since there is less deviation from the centre line in the data from the experiment.

For future research, it remains to be investigated to what extent the free surface at the top of the fluid domain plays a role in system dynamics. Furthermore, an open question is that of the role of imperfections in the mass distribution within the solid, of the surface roughness of the solid and whether these can explain larger horizontal displacements and rotation observed for example with the POM particle in [HTR20].

\section{Acknowledgements}

HvW and TR acknowledge support by the Deutsche Forschungsgemeinschaft (DFG, German Research Foundation) - 314838170, GRK 2297 MathCoRe. TR further acknowledges support by the Federal Ministry of Education and Research of Germany (project number 05M16NMA).

\section{References}

[Age+19] C. Ager, B. Schott, A. T. Vuong, A. Popp and W. A. Wall. 'A consistent approach for fluid-structure-contact interaction based on a porous flow model for rough surface contact'. In: Internat. J. Numer. Methods Engrg. 119.13 (2019), pp. 1345-1378. DOI: 10.1002/nme.6094.

[ASW19] C. Ager, A. Seitz and W. A. WALl. A consistent and comprehensive computational approach for general FluidStructure-Contact Interaction problems. 23rd May 2019. arXiv: 1905.09744.

[AC91] P. AlarT and A. Curnier. 'A mixed formulation for frictional contact problems prone to Newton like solution methods'. In: Comput. Methods Appl. Mech. Eng. 92.3 (Nov. 1991), pp. 353-375. DOI: 10.1016/0045-7825(91) 90022-X.

[AmR04] M. L. Anderson, P. H. Mott and C. M. Roland. 'The Compression of Bonded Rubber Disks'. In: Rubber Chem. Technol. 77.2 (May 2004), pp. 293-302. DOI: 10.5254/1.3547824.

[Baa01] F. P. T. BAAiJens. 'A fictitious domain/mortar element method for fluid-structure interaction'. In: Internat. J. Numer. Methods Fluids 35.7 (2001), pp. 743-761. DOI: 10.1002/1097-0363(20010415) $35: 7<743:$ : AID FLD109>3.0.CO;2-A.

[BM84] I. BABUŠKA and A. Miller. 'The post-processing approach in the finite element method. I: Calculations of displacements, stresses and other higher derivatives of the displacements'. In: Inter. J. Numer. Meth. Engrg. (1984). DOI: $10.1002 / \mathrm{nme} .1620200610$.

[BB01] R. BECKER and M. BRAACK. 'A finite element pressure gradient stabilization for the Stokes equations based on local projections'. In: Calcolo 38.4 (Dec. 2001), pp. 173-199. DOI: 10.1007/s10092-001-8180-4.

[BDM99] C. Bernardi, M. Dauge and Y. Maday. Spectral Methods for Axisymetric Domain. Series in Applied Mathematics. Paris: Gauthier-Villars, 1999. ISBN: 2-84299-070-6.

[BR06] M. BraAcK and T. Richter. 'Local Projection Stabilization for the Stokes System on Anisotropic Quadrilateral Meshes'. In: Numerical Mathematics and Advanced Applications. Proceedings of ENUMATH 2005 the 6th European Conference on Numerical Mathematics and Advanced Applications, Santiago de Compostela, Spain, July 2005. Ed. by A. De Castro, D. Gómez, P. Quintela and P. Salgado. Springer, 2006. Doi: 10.1007/9783-540-34288-5_75.

[Bre61] H. BREnNer. 'The slow motion of a sphere through a viscous fluid towards a plane surface'. In: Chem. Eng. Sci. 16.3-4 (Dec. 1961), pp. 242-251. DOI: 10.1016/0009-2509(61)80035-3.

[Bur10] E. Burman. 'Ghost penalty'. In: C.R. Math. 348.21-22 (Nov. 2010), pp. 1217-1220. DOI: 10.1016/j.crma.2010. 10.006 .

[Bur+19] E. Burman, M. A. Fernández, S. Frei and F. M. Gerosa. 3D-2D Stokes-Darcy coupling for the modelling of seepage with an application to fluid-structure interaction with contact. 2019. arXiv: 1912.08503.

[BFM19] E. Burman, S. Frei and A. Massing. Eulerian time-stepping schemes for the non-stationary Stokes equations on time-dependent domains. 7th Oct. 2019. arXiv: 1910.03054v1.

[BFF20] E. Burman, M. A. FERnÁndez and S. Frei. 'A Nitsche-based formulation for fluid-structure interactions with contact'. In: ESAIM Math. Model. Numer. Anal. 54.2 (2020), pp. 531-564. DOI: 10.1051/m2an/2019072. 
[BF14] E. Burman and M. A. Fernández. 'An unfitted Nitsche method for incompressible fluid-structure interaction using overlapping meshes'. In: Comput. Methods Appl. Mech. Eng. 279 (2014), pp. 497-514. DOI: 10.1016/j .cma. 2014.07 .007$.

[CMM08] G.-H. Cottet, E. Maitre and T. Milcent. 'Eulerian formulation and level set models for incompressible fluidstructure interaction'. In: ESAIM Math. Model. Numer. Anal. 42.3 (Apr. 2008), pp. 471-492. DOI: 10.1051/m2an: 2008013.

[CF15] S. CourT and M. FourniÉ. 'A fictitious domain finite element method for simulations of fluid-structure interactions: The Navier-Stokes equations coupled with a moving solid'. In: J. Fluids. Struct. 55 (May 2015), pp. 398-408. DOI: $10.1016 / j \cdot j$ fluidstructs.2015.03.013.

[CFL13] S. Court, M. Fournié and A. Lozinski. 'A fictitious domain approach for the Stokes problem based on the extended finite element method'. In: Internat. J. Numer. Methods Fluids 74.2 (Aug. 2013), pp. 73-99. DOI: 10.1002/fld.3839.

[DSH86] R. H. Davis, J.-M. Serayssol and E. J. Hinch. 'The elastohydrodynamic collision of two spheres'. In: J. Fluid Mech. 163 (Feb. 1986), pp. 479-497. DOI: 10.1017/s0022112086002392.

[DGH82] J. Donea, S. Giuliani and J. Halleux. 'An arbitrary Lagrangian-Eulerian finite element method for transient dynamic fluid-structure interactions'. In: Comput. Methods Appl. Mech. Eng. 33.1-3 (Sept. 1982), pp. 689-723. DOI: $10.1016 / 0045-7825$ (82) 90128-1.

[Dun06] T. DunNE. 'An Eulerian approach to fluid-structure interaction and goal-oriented mesh adaptation'. In: Int. J. Numer. Methods Fluids 51.9-10 (2006), pp. 1017-1039. DOI: 10.1002/fld.1205.

[FR20] L. FAiler and T. Richter. 'A parallel Newton multigrid framework for monolithic fluid-structure interactions'. In: J. Sci. Comput. 82.2 (2020). DOI: 10.1007/s11081-020-09498-8.

[Fei03a] E. Feireisl. 'On the Motion of Rigid Bodies in a Viscous Compressible Fluid'. In: Arch. Ration. Mech. Anal. 167.4 (May 2003), pp. 281-308. DOI: 10.1007/s00205-002-0242-5.

[Fei03b] E. FeIreisl. 'On the motion of rigid bodies in a viscous incompressible fluid'. In: Nonlinear Evolution Equations and Related Topics. Birkhäuser Basel, 2003, pp. 419-441. DOI: 10.1007/978-3-0348-7924-8_23.

[Fre16] S. Frei. 'Eulerian finite element methods for interface problems and fluid-structure interactions'. PhD thesis. Ruprecht-Karls-Universität Heidelberg, 23rd Aug. 2016. DOI: 10.11588/heidok.00021590.

[FRW16] S. Frei, T. Richter and T. Wick. 'Long-term simulation of large deformation, mechano-chemical fluid-structure interactions in ALE and fully Eulerian coordinates'. In: J. Comput. Phys. 321 (2016), pp. 874-891. DOI: 10.1016/ $j \cdot j c p .2016 .06 .015$.

[Fre19] S. Frei. 'An edge-based pressure stabilization technique for finite elements on arbitrarily anisotropic meshes'. In: Internat. J. Numer. Methods Fluids 89.10 (2019), pp. 407-429. DOI: 10.1002/fld.4701.

[FR14] S. Frei and T. RichteR. 'A locally modified parametric finite element method for interface problems'. In: SIAM J. Numer. Anal. 52.5 (2014), pp. 2315-2334. DOI: 10.1137/130919489.

[FR17] S. Frei and T. Richter. 'A second order time-stepping scheme for parabolic interface problems with moving interfaces'. In: ESAIM Math. Model. Numer. Anal. 51.4 (2017), pp. 1539-1560. DOI: 10.1051/m2an/2016072.

[Bec+] R. Becker, M. BraAck, D. Meidner, T. Richter and B. Vexler. The finite element toolkit Gascoigne3d. url: http://www.gascoigne.de.

[GH14] D. GÉrard-Varet and M. Hillairet. 'Existence of Weak Solutions Up to Collision for Viscous Fluid-Solid Systems with Slip'. In: Commun. Pure Appl. Math. 67.12 (May 2014), pp. 2022-2076. DoI: 10.1002/cpa.21523.

[GHW15] D. Gérard-VAREt, M. Hillairet and C. WANG. 'The influence of boundary conditions on the contact problem in a 3D Navier-Stokes flow'. In: J. Math. Pures Appl. 103.1 (Jan. 2015), pp. 1-38. DOI: 10.1016/j.matpur.2014.03.005.

[GW08] A. Gerstenberger and W. A. WALL. 'An eXtended Finite Element Method/Lagrange multiplier based approach for fluid-structure interaction'. In: Comput. Methods Appl. Mech. Engrg. 197.19 (Mar. 2008), pp. 1699-1714. DOI: $10.1016 / j . c m a .2007 .07 .002$.

[Glo+99] R. Glowinski, T.-W. Pan, T. I. Hesla and D. D. Joseph. 'A distributed Lagrange multiplier/fictitious domain method for particulate flows'. In: Int. J. Multiph. Flow 25.5 (Aug. 1999), pp. 755-794. DOI: 10.1016/s0301$9322(98) 00048-2$.

[GH16] C. Grandmont and M. Hillairet. 'Existence of Global Strong Solutions to a Beam-Fluid Interaction System'. In: Arch. Ration. Mech. Anal. 220.3 (Jan. 2016), pp. 1283-1333. DOI: 10.1007/s00205-015-0954-y.

$[\mathrm{Gra}+20] \quad$ G. Gravina, S. Schwarzacher, O. SoučEK and K. Tưma. Contactless rebound of elastic bodies in a viscous incompressible fluid. 3rd Nov. 2020. arXiv: 2011.01932v1.

[GO17] J. GuZMÁn and M. Olshanskir. 'Inf-sup stability of geometrically unfitted Stokes finite elements'. In: Math. Comp. 87.313 (Dec. 2017), pp. 2091-2112. DOI: 10.1090/mcom/3288.

[Hag20] T. Hagemeier. Particle Settling-Transitional Regime. Version 1. 1st Sept. 2020. DOI: 10.17632/mf27c92nc3.1.

[HTR20] T. HAGEMeier, D. ThÉvenin and T. Richter. Settling of spherical, non-wetting particles in a high viscous fluid. 4th Sept. 2020. arXiv: 2009.02250v1.

[HH03] P. HANSBO and J. Hermansson. 'Nitsche's method for coupling non-matching meshes in fluid-structure vibration Problems'. In: Comput. Mech. 32.1-2 (Sept. 2003), pp. 134-139. DOI: 10.1007/s00466-003-0467-7.

[HP17] F. Hecht and O. Pironneau. 'An energy stable monolithic Eulerian fluid-structure finite element method'. In: Internat. J. Numer. Methods Fluids 85.7 (May 2017), pp. 430-446. DOI: 10.1002/fld. 4388. 
[HT09] M. Hillairet and T. Takahashi. 'Collisions in Three-Dimensional Fluid Structure Interaction Problems'. In: SIAM J. Math. Anal. 40.6 (Jan. 2009), pp. 2451-2477. DOI: 10.1137/080716074.

[HLZ81] T. J. R. Hughes, W. K. Liu and T. K. Zimmermann. 'Lagrangian-Eulerian finite element formulations for incompressible viscous flows'. In: Comp. Meth. Appl. Mech. Engrg. 29.3 (Dec. 1981), pp. 329-349. DoI: 10.1016/ 0045-7825 (81) 90049-9.

[JlL15] A. Johansson, M. G. Larson and A. LogG. 'High order cut finite element methods for the Stokes problem'. In: Adv. Model. Simul. Eng. Sci. 2.1 (Sept. 2015). DOI: 10.1186/s40323-015-0043-7.

[Joh+17] V. John, A. Linke, C. Merdon, M. Neilan and L. G. Rebholz. 'On the divergence constraint in mixed finite element methods for incompressible flows'. In: SIAM Rev. 59.3 (Jan. 2017), pp. 492-544. DOI: 10.1137/15m1047696.

[LCB06] A. Legay, J. Chessa and T. Belytschko. 'An Eulerian-Lagrangian method for fluid-structure interaction based on level sets'. In: Comput. Methods Appl. Mech. Engrg. 195.17-18 (Mar. 2006), pp. 2070-2087. DOI: 10.1016/j . cma.2005.02.025.

[Leh16] C. Lehrenfeld. 'High order unfitted finite element methods on level set domains using isoparametric mappings'. In: Comput. Methods Appl. Mech. Engrg. 300 (Mar. 2016), pp. 716-733. DOI: 10.1016/j.cma.2015.12.005.

[LO19] C. Lehrenfeld and M. A. Olshanskit. 'An Eulerian finite element method for PDEs in time-dependent domains'. In: ESAIM Math. Model. Numer. Anal. 53.2 (Mar. 2019), pp. 585-614. DOI: 10.1051/m2an/2018068.

[Man+15] H. Mann, P. Mueller, T. Hagemeier, C. Roloff, D. Thévenin and J. Tomas. 'Analytical description of the unsteady settling of spherical particles in Stokes and Newton regimes'. In: Granul. Matter 17.5 (Sept. 2015), pp. 629-644. DOI: $10.1007 / \mathrm{s} 10035-015-0584-\mathrm{y}$.

[xfem1.3] ngsxfem: An add-on to NGSolve for unfitted finite element discretizations. Version 1.3.2008. 2020. URL: https: //github.com/ngsxfem/ngsxfem.

[Nit71] J. Nitsche. 'Über ein Variationsprinzip zur Lösung von Dirichlet-Problemen bei Verwendung von Teilräumen, die keinen Randbedingungen unterworfen sind'. In: Abh. Math. Semin. Univ. Hambg. 36.1 (July 1971), pp. 9-15. DOI: $10.1007 / \mathrm{bf} 02995904$.

[Pre18] J. Preuss. 'Higher order unfitted isoparametric space-time FEM on moving domains'. MA thesis. Georg-August Universität Göttingen, 2018. URL: http://num.math.uni-goettingen.de/ lehrenfeld/sections/pubs_src/ Pre18_Ma.pdf.

[Ric17] T. RICHTER. Fluid-structure Interactions. Models, Analysis and Finite Elements. Vol. 118. Lecture Notes in Computational Science and Engineering. Springer, 2017. DOI: 10.1007/978-3-319-63970-3.

[Ric13] T. Richter. 'A Fully Eulerian Formulation for Fluid-Structure Interactions'. In: J. Comput. Phys. 233 (2013), pp. 227-240. DOI: $10.1016 / j \cdot j c p .2012 .08 .047$.

[ST08] S. SAthe and T. E. Tezduyar. 'Modeling of fluid-structure interactions with the space-time finite elements: contact problems'. In: Comput. Mech. 43.1 (May 2008), pp. 51-60. DOI: 10.1007/s00466-008-0299-6.

[Sch97] J. SchöвERL. 'NETGEN an advancing front 2D/3D-mesh generator based on abstract rules'. In: Comput. Vis. Sci. 1.1 (July 1997), pp. 41-52. DOI: 10.1007/s007910050004.

[Sch14] J. SchöBerL. C++11 implementation of finite elements in NGSolve. ASC Report No. 30/2014. Tech. rep. Institute for Analysis and Scientific Computing, TU Wien, 26th Sept. 2014. URL: http://www . asc. tuwien . ac . at/ $\sim$ schoeberl/wiki/publications/ngs-cpp11.pdf.

[SAW19] B. Sснотt, C. Ager and W. A. Wall. 'A monolithic approach to fluid-structure interaction based on a hybrid Eulerian-ALE fluid domain decomposition involving cut elements'. In: Internat. J. Numer. Methods Engrg. (Mar. 2019). DOI: $10.1002 / \mathrm{nme} \cdot 6047$.

[TH06] S. TureK and J. Hron. 'Proposal for Numerical Benchmarking of Fluid-Structure Interaction between an Elastic Object and Laminar Incompressible Flow'. In: Lecture Notes in Computational Science and Engineering. Springer Berlin Heidelberg, 2006, pp. 371-385. DOI: 10.1007/3-540-34596-5_15.

[Wah+20] H. von Wahl, T. Richter, S. Frei and T. HAGemeier. Falling balls in a viscous fluid with contact: Comparing numerical simulations with experimental data. Version 1. 17th Nov. 2020. DOI: 10.5281/zenodo.3989604.

[WRL20] H. von Wahl, T. Richter and C. Lehrenfeld. An unfitted Eulerian finite element method for the time-dependent Stokes problem on moving domains. 6th Feb. 2020. arXiv: 2002.02352v1.

[Wah+19] H. von Wahl, T. Richter, C. Lehrenfeld, J. Heiland and P. Minakowski. 'Numerical benchmarking of fluidrigid body interactions'. In: Comput. \& Fluids 193, 104290 (Oct. 2019). DOI: 10.1016/j .compfluid.2019.104290.

[WT06] D. WAN and S. TUREK. 'Direct numerical simulation of particulate flow via multigrid FEM techniques and the fictitious boundary method'. In: Internat. J. Numer. Methods Fluids 51.5 (2006), pp. 531-566. DOI: 10.1002/fld. 1129.

[ZAV20] S. Zonca, P. F. Antonietti and C. Vergara. A Polygonal Discontinuous Galerkin formulation for contact mechanics in fluid-structure interaction problems. MOX-Report No. 26/2020. MOX, Dipartimento di Matematica Politecnico di Milano. 5th May 2020. uRL: https://www. mate.polimi.it/biblioteca/add/qmox/26-2020.pdf (visited on $02 / 11 / 2020$ ). 


\section{A. Computational test cases}

We define two simplified test cases. This is intended make it easier for others to reproduce the presented results using different methods and/or implementations.

\section{A.1. Stationary flow test}

For this stationary test, we modify the Rubber22 set up. The sphere is fixed at $c_{\mathcal{S}}=(0,0,0.1)$, i.e., the centre of the cylinder. We impose an inflow boundary condition $\boldsymbol{u}=-0.01\left(1-\left(\boldsymbol{x}_{1}^{2}+x_{2}^{2}\right) / R^{2}\right)$ on $\Gamma_{\text {top }}$, no-slip $\boldsymbol{u}=0$ on $\Gamma_{\text {wall }} \cup \mathcal{I}$ and a homogeneous Neumann boundary condition $\boldsymbol{\sigma}(\boldsymbol{u}, p) \boldsymbol{n}=\mathbf{0}$ on $\Gamma_{\text {bottom }}$.

We consider the stationary Navier-Stokes problem on this domain. As reference quantities we take the vertical stress acting on the sphere, i.e., testing the reduced formulation in (3.5) with the non-conforming, continuous test-functions $\widehat{\boldsymbol{w}}=(0,1)^{T}$ on $\mathcal{I}$ and $\mathbf{0}$ on $\Gamma$ respectively.

We compute the problem based on the discretisations discussed in subsubsection 4.1.1 and subsubsection 4.1.2. The results can be seen in Table 6 .

\begin{tabular}{|c|c|c|c|c|}
\hline \multicolumn{4}{|c|}{ Discretisation } & \multirow{2}{*}{$\begin{array}{l}\text { Results } \\
\boldsymbol{F}_{z}\end{array}$} \\
\hline Method & {$\left[h_{\min }, h_{\max }\right]$} & dof & nze & \\
\hline \multirow[t]{2}{*}{ ALE } & $\begin{array}{l}{[0.00030,0.0049]} \\
{[0.00015,0.0024]} \\
{[0.00008,0.0012]}\end{array}$ & $\begin{array}{r}6.9 \\
26.7 \\
104.9\end{array}$ & $\begin{array}{l}0.310 \\
1.238 \\
4.946\end{array}$ & $\begin{array}{l}-4.43234 \cdot 10^{-5} \\
-4.42992 \cdot 10^{-5} \\
-4.42975 \cdot 10^{-5}\end{array}$ \\
\hline & & \multicolumn{2}{|c|}{$\begin{array}{l}\text { extrapolate } \\
\text { order (in h) }\end{array}$} & $\begin{array}{l}-4.42974 \cdot 10^{-5} \\
3.8\end{array}$ \\
\hline CutFEM & $\begin{array}{l}{[0.0020,0.008]} \\
{[0.0010,0.004]} \\
{[0.0005,0.002]}\end{array}$ & $\begin{array}{r}11.9 \\
41.3 \\
151.4\end{array}$ & $\begin{array}{l}0.361 \\
1.241 \\
4.525\end{array}$ & $\begin{array}{l}-4.40254 \cdot 10^{-5} \\
-4.42355 \cdot 10^{-5} \\
-4.42919 \cdot 10^{-5}\end{array}$ \\
\hline
\end{tabular}

Table 6.: Resulting reference quantities for the stationary test scenario. ${ }^{5}$

\section{A.2. Non-stationary flow test with prescribed motion}

As a second test case we keep the Rubber22 set-up as the basis. The material parameters and the cylinder boundary conditions are as before, i.e., we consider no-slip on $\Gamma_{\text {wall }} \cup \Gamma_{\text {bottom }}$ and a free-slip condition on $\Gamma_{\text {top }}$. We prescribe the motion of the sphere as follows. Over the time-interval $I=[0,20]$, the sphere is located at

$$
c_{\mathcal{S}}=(0,0, d(t)) \quad \text { with } \quad d(t)=0.1+0.05 \cos (0.1 \pi t) .
$$

The boundary condition on the interface is accordingly set to $\boldsymbol{u}=\left(0,0, \partial_{t} d(t)\right)$. Quantities of interest are the maximal value over time of the $z$-component of the force functional in the reduced formulation.

We compute this again using the reduced formulation with the rigid body discretisations. The quantitative results can be seen in Table 7 while the force functional is shown over time in Figure 7.

\begin{tabular}{|c|c|c|c|c|c|c|}
\hline \multicolumn{5}{|c|}{ Discretisation } & \multicolumn{2}{|c|}{ Results } \\
\hline Method & {$\left[h_{\min }, h_{\max }\right]$} & $\Delta t$ & dof & nze & $\boldsymbol{F}_{z, \max }$ & $t_{z, \max }$ \\
\hline \multirow[t]{3}{*}{ ALE } & {$[0.00030,0.0099]$} & $1 / 5$ & 11.1 & 0.49 & $1.01838 \cdot 10^{-4}$ & 4.10734691 \\
\hline & {$[0.00015,0.0050]$} & $1 / 20$ & 25.0 & 1.10 & $1.01748 \cdot 10^{-4}$ & 4.11356325 \\
\hline & {$[0.00008,0.0025]$} & $1 / 80$ & 58.7 & 2.61 & $1.01720 \cdot 10^{-4}$ & 4.10670436 \\
\hline \multirow[t]{3}{*}{ CutFEM $^{4}$} & {$[0.0020,0.008]$} & $1 / 25$ & 11.9 & 0.361 & $1.01700 \cdot 10^{-4}$ & 4.153 \\
\hline & {$[0.0010,0.004]$} & $1 / 50$ & 41.2 & 1.240 & $1.01395 \cdot 10^{-4}$ & 4.138 \\
\hline & {$[0.0005,0.002]$} & $1 / 100$ & 151.3 & 4.526 & $1.01671 \cdot 10^{-4}$ & 4.111 \\
\hline
\end{tabular}

Table 7.: Resulting reference quantities for the non-stationary moving domain test scenario. ${ }^{5}$ 


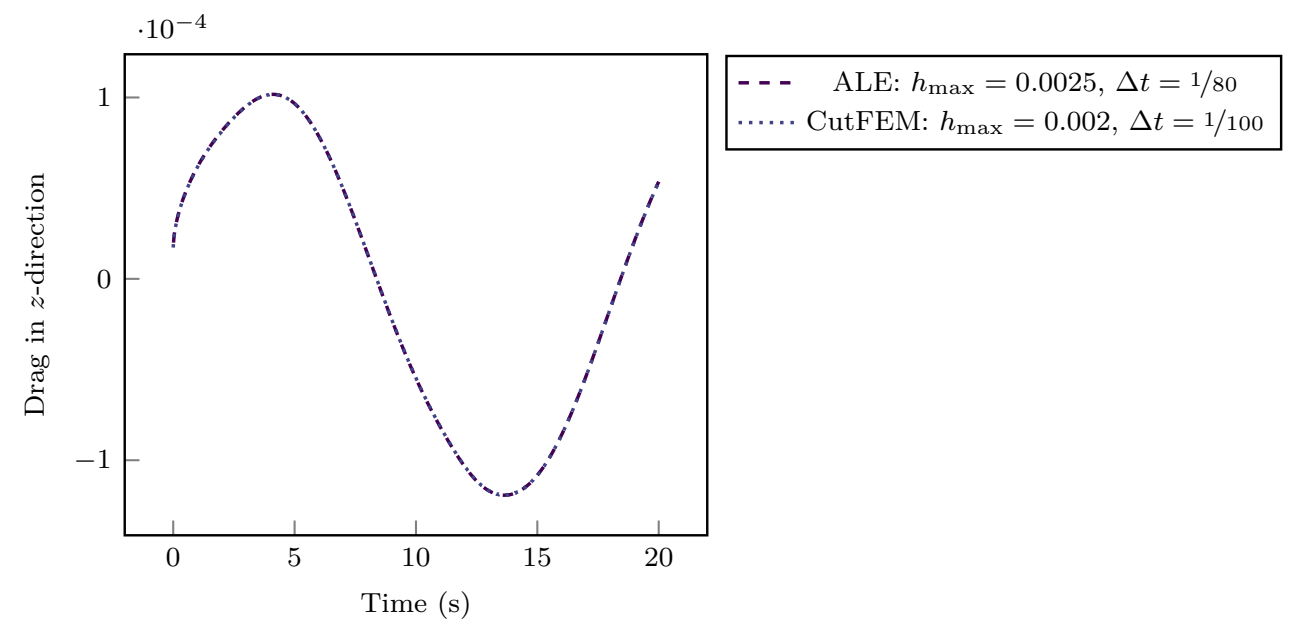

Figure 7.: Force functionals acting on the sphere with prescribed motion over time. 